\title{
Hydrogen Sulfide-Mediated Polyamines and Sugar Changes Are Involved in Hydrogen Sulfide-Induced Drought Tolerance in Spinacia oleracea Seedlings
}

\author{
Juan Chen ${ }^{1 \dagger}$, Yu-Ting Shang ${ }^{1 \dagger}$, Wen-Hua Wang ${ }^{2}$, Xi-Yan Chen ${ }^{3}$, En-Ming He'2, \\ Hai-Lei Zheng ${ }^{4}$ and Zhouping Shangguan ${ }^{1 *}$ \\ 'State Key Laboratory of Soil Erosion and Dryland Farming on the Loess Plateau, Northwest A\&F University, Yangling, \\ China, ${ }^{2}$ Fujian Key Laboratory of Subtropical Plant Physiology and Biochemistry, Fujian Institute of Subtropical Botany, \\ Xiamen, China, ${ }^{3}$ College of Life Science, Northwest A\&F University, Yangling, China, ${ }^{4}$ Key Laboratory of the Ministry of \\ Education for Coastal and Wetland Ecosystem, College of the Environment and Ecology, Xiamen University, Xiamen, China
}

OPEN ACCESS

Edited by:

Maren Müller,

University of Barcelona, Spain

Reviewed by:

Vasileios Fotopoulos,

Cyprus University of Technology,

Cyprus

Ravi Valluru,

International Maize and Wheat Improvement Center, Mexico

${ }^{*}$ Correspondence:

Zhouping Shangguan

shangguan@ms.iswc.ac.cn

${ }^{\dagger}$ These authors have contributed equally to this work.

Specialty section:

This article was submitted to Plant Physiology,

a section of the journal Frontiers in Plant Science

Received: 26 April 2016 Accepted: 21 July 2016 Published: 04 August 2016

Citation:

Chen J, Shang $Y-T$, Wang $W-H$, Chen $X-Y$, He E-M, Zheng $H-L$ and Shangguan $Z$ (2016) Hydrogen Sulfide-Mediated Polyamines and Sugar Changes Are Involved in Hydrogen Sulfide-Induced Drought

Tolerance in Spinacia oleracea Seedlings. Front. Plant Sci. 7:1173. doi: 10.3389/fpls.2016.01173
Hydrogen sulfide $\left(\mathrm{H}_{2} \mathrm{~S}\right)$ is a newly appreciated participant in physiological and biochemical regulation in plants. However, whether $\mathrm{H}_{2} \mathrm{~S}$ is involved in the regulation of plant responses to drought stress remains unclear. Here, the role of $\mathrm{H}_{2} \mathrm{~S}$ in the regulation of drought stress response in Spinacia oleracea seedlings is reported. First, drought stress dramatically decreased the relative water content (RWC) of leaves, photosynthesis, and the efficiency of PSII. Moreover, drought caused the accumulation of ROS and increased the MDA content. However, the application of NaHS counteracted the drought-induced changes in these parameters. Second, $\mathrm{NaHS}$ application increased the water and osmotic potential of leaves. Additionally, osmoprotectants such as proline and glycinebetaine (GB) content were altered by NaHS application under drought conditions, suggesting that osmoprotectant contributes to $\mathrm{H}_{2} \mathrm{~S}$-induced drought resistance. Third, the levels of soluble sugars and polyamines (PAs) were increased differentially by NaHS application in S. oleracea seedlings. Moreover, several genes related to PA and soluble sugar biosynthesis, as well as betaine aldehyde dehydrogenase (SoBADH), choline monooxygenase (SoCMO), and aquaporin $(\mathrm{SoPIP1} ; 2)$, were up-regulated by $\mathrm{H}_{2} \mathrm{~S}$ under drought stress. These results suggest that $\mathrm{H}_{2} \mathrm{~S}$ contributes to drought tolerance in $\mathrm{S}$. oleracea through its effect on the biosynthesis of PAs and soluble sugars. Additionally, GB and trehalose also play key roles in enhancing $S$. oleracea drought resistance.

Keywords: hydrogen sulfide, drought, soluble sugar, polyamines, water relations, Spinacia oleracea

\section{INTRODUCTION}

Drought induces water scarcity and osmotic stresses that restrict the growth and development of plants because water absorption by plant roots from the soil is inadequate to satisfy plant transpiration demand (Blum, 1996). Additionally, leaf cell turgor is significantly lessened by water deficiency, which in turn restricts cell expansion, the increase of leaf area, and photosynthesis, thus inhibiting the accumulation of biomass (Chaves et al., 2003). An effective strategy for plant drought 
adaptability has been to inhibit water scarcity caused by transpiration, which saves soil moisture and allows plants to sustain sufficient water content to maintain key physiological processes (Yoo et al., 2010).

During water deficiency and other osmotic stresses, the accumulation of compatible low-molecular-weight osmoprotectants, such as soluble sugars (including glucose, fructose, and sucrose), proline, sugar alcohols, special amino acids and glycinebetaine (GB) may confer stress tolerance to plants (García-Mata and Lamattina, 2001; Rivero et al., 2014). Additionally, the composition and content of the osmoprotectants in stressed plants can vary considerably depending on the species and environmental conditions (Rivero et al., 2014). Moreover, polyamines (PAs), including putrescine (Put), spermidine (Spd) and spermine (Spm), are ubiquitous biological organic-amines involved in plant growth, development and the response to biotic and abiotic stresses (Takahashi and Kakehi, 2010). When plants are exposed to stress conditions such as drought, heat, chilling, or salinity, PA levels significantly increase and become one of the most prominent metabolic features (Takahashi and Kakehi, 2010; Tiburcio et al., 2014). These changes are caused mainly by the biosynthesis of PAs, PA oxidation, and/or interactions with other pathways under stress conditions (Tiburcio et al., 2014). The PA metabolic pathway mediates nitrogen or carbon metabolism and interacts with other metabolites, such as stress-protective compounds including signaling molecules and hormones (Moschou et al., 2012; Tiburcio et al., 2014). As an example, the abscisic acid (ABA)-induced accumulation of PAs enhances PA oxidation, which in turn enacts protection against such stresses as against drought in plants (Toumi et al., 2010). Additionally, many previous studies have shown that PAs play an important role in salt tolerance and drought resistance in plants (Alcázar et al., 2010b; Yin et al., 2013, 2015). Under drought stress, plants with high PA contents exhibit strong adaptability, and exogenous PAs alleviate drought stress. All these results demonstrate that PA levels are positively correlated to drought resistance in plants (Capell et al., 2004). Recently, much research has indicated that the overexpression of PA biosynthesis-related genes increases PA content and improves drought tolerance or salt stress (Capell et al., 2004; Wen et al., 2008; Alcázar et al., 2010a). Moreover, knockout/knock-down mutants whose capacity to synthesize PAs were abolished or limited were more sensitive to drought or salt stress than wild-type plants (Kasinathan and Wingler, 2004). Together, these studies indicate that PAs play a vital role in regulating drought tolerance and salt resistance in plants.

The small bioactive gaseous hydrogen sulfide $\left(\mathrm{H}_{2} \mathrm{~S}\right)$ exhibits both physical and functional similarities with other gasotransmitters such as carbon monoxide (CO) and nitric oxide (NO) and has been shown to be involved in different physiological processes in animals (Hosoki et al., 1997; Zhao et al., 2001; Wang, 2002; Li et al., 2006; Yang et al., 2008). $\mathrm{NaHS}$, a donor of $\mathrm{H}_{2} \mathrm{~S}$, has been used to study the physiological function of $\mathrm{H}_{2} \mathrm{~S}$ in animals and plants (Hosoki et al., 1997; Zhang et al., 2008; García-Mata and Lamattina, 2010). Recently, much of this research has focused on the physiological function of $\mathrm{H}_{2} \mathrm{~S}$ in plants. Specifically, $\mathrm{H}_{2} \mathrm{~S}$ promotes seed germination, protects against copper-induced oxidative damage, counteracts chlorophyll loss, and alleviates oxidative damage from water scarcity in sweet potato leaves (Zhang et al., 2008, 2009). Additionally, boron, salinity and aluminum toxicities were alleviated by $\mathrm{H}_{2} \mathrm{~S}$ treatment (Wang et al., 2010, 2012; Zhang et al., 2010b; Chen et al., 2013, 2015a). Furthermore, low concentrations of $\mathrm{H}_{2} \mathrm{~S}$ regulated flower senescence and promoted the embryonic root length of Pisum sativum (Li et al., 2010; Zhang et al., 2011). In addition, García-Mata and Lamattina (2010) reported that $\mathrm{H}_{2} \mathrm{~S}$ regulated the ATP-binding cassette $(\mathrm{ABC})$ transporter in guard cells and then caused stomatal closure; these processes are closely connected to the ABA signaling pathway. Our previous study also showed that $\mathrm{H}_{2} \mathrm{~S}$ promoted iron availability in Zea mays seedlings and enhanced photosynthesis in S. oleracea seedlings (Chen et al., 2011, 2015b). Moreover, many previous studies have shown that $\mathrm{H}_{2} \mathrm{~S}$ can effectively improve drought tolerance in many different plants (Zhang et al., 2010a; Shan et al., 2011; Jin et al., 2013). For example, Ziogas et al. (2015) reported that $\mathrm{H}_{2} \mathrm{~S}$ pre-treatment led to the post-translational modification (PTM) of proteins under drought stress in citrus plants. Additionally, Li et al. (2015) showed that $\mathrm{H}_{2} \mathrm{~S}$ alleviated drought-induced PSII damage due to fast D1 protein turnover. A previous study also showed that $\mathrm{H}_{2} \mathrm{~S}$ regulated the expression of drought-related genes including $D R E B$ and RD29A (Jin et al., 2011). The aforementioned studies focused on only the protection against drought-induced oxidative damage $\mathrm{H}_{2} \mathrm{~S}$ offered via the enhancement of non-enzymatic and enzymatic antioxidant capacities, the reduction of evaporation, or the control of stomatal conductance in plants. However, the detailed mechanisms of the effects of $\mathrm{H}_{2} \mathrm{~S}$ on drought stress remain unclear, especially with respect to physiological processes in plants.

Because PAs and soluble sugars particularly enhance drought tolerance, the question of whether PAs and soluble sugars are involved in $\mathrm{H}_{2} \mathrm{~S}$-induced drought tolerance needs to be addressed. Recent studies have shown that the bioactive free radical molecule $\mathrm{NO}$ and PAs share certain overlapping physiological roles in plants (Yamasaki and Cohen, 2006). The synthesis of NO was induced by PAs in Arabidopsis, which indicates that $\mathrm{NO}$ might contribute to PA signaling during stress (Tun et al., 2006). However, whether a low concentration of $\mathrm{H}_{2} \mathrm{~S}$ similar to that of NO has an effect on PA levels and further enhances the resistance of plants to stress remains unclear.

In this study, we present important data that reveals a novel effect of $\mathrm{H}_{2} \mathrm{~S}$ in plant physiology, more specifically on drought stress. These results support the suggestion that $\mathrm{H}_{2} \mathrm{~S}$ significantly enhances the tolerance of plants to drought stress through its effects on PA and soluble sugar contents, which change the expression levels of genes associated with PAs and sugar biosynthesis.

\section{MATERIALS AND METHODS}

\section{Plant Culture and Treatment}

Seeds of Spinacia oleracea were first sterilized by immersion in $75 \%$ ethanol for $3 \mathrm{~min}$ followed by $10 \mathrm{~min}$ in a $10 \%$ 
sodium hypochlorite solution. Next, seeds were washed with distilled water and germinated in a soil/vermiculite (1:1) mixture. Subsequently, 1-week-old seedlings were transferred to plastic pots $(15 \mathrm{~cm} \times 15 \mathrm{~cm} ; 25$ seedlings per pot) filled with a soil/vermiculite (1:1) mixture The plants were then grown in a controlled growth chamber with a light/dark regime of 10/14 h, a relative humidity of $80 \%$, a temperature of $21 / 27^{\circ} \mathrm{C}$, and a photosynthetically active radiation (PAR) of $190 \mu \mathrm{mol} \mathrm{m}^{-2} \mathrm{~s}^{-1}$ and were watered with a $1 / 2$ strength Hoagland solution every other days.

$\mathrm{NaHS}$ was used as the exogenous $\mathrm{H}_{2} \mathrm{~S}$ donor as described by Hosoki et al. (1997). Six-week-old seedlings were treated with the $1 / 2$ strength Hoagland solution containing $100 \mu \mathrm{M}$ NaHS. The same volume $1 / 2$ strength Hoagland solution was used as the control. For drought treatment, 6-week-old plants subjected to drought stress, were grown in pots by withholding water for 8 days, and then re-watered until the substrate was saturated. Treated leaves were sampled after 0,5 , and 8 days of drought stress and after 1 and 4 days of re-watering. All samples were frozen rapidly in liquid nitrogen and stored at $-80^{\circ} \mathrm{C}$.

\section{Measurement of Soil Water Content, Plant Survival, Leaf Relative Water Content, and Detached Leaf Water Loss}

Drought stress was imposed by withholding water from containers of soilless media (with dry weight of $58.6 \pm 5.4 \mathrm{~g}$ ) containing 25 plants (6-week-old). The containers were irrigated with water to saturation and weighed at the start of the drought stress treatment (initial weight) and then periodically throughout the treatment period. Relative soil water content (SWC) was calculated as follows: (final fresh weight-dry weight)/(initial weight-dry weight $) \times 100$. After 8 days of drought, the plants were re-watered, and the plant survival was measured 4 days after re-watering (Yoo et al., 2010).

In a separate experiment, the relative water content (RWC) of fully expanded leaves was assessed in 6-week-old plants growing at different relative SWC. Leaves were cut and immediately weighed to obtain the leaf FW. The leaves were then placed into small bottles filled with distilled water for $24 \mathrm{~h}$, blotted up to remove excess water, and then weighed to obtain the leaf turgid weight (TW). The leaves were then dried to a constant weight at $70^{\circ} \mathrm{C}$ and reweighed to obtain the leaf dry weight (DW). The leaf RWC was calculated as follows: $(\mathrm{FW}-\mathrm{DW}) /(\mathrm{TW}-\mathrm{DW}) \times 100$ (Yoo et al., 2010).

The detached leaves were treated with different concentrations of NaHS $(0,10,100,500$, and $1000 \mu \mathrm{M})$ for $3 \mathrm{~h}$ and the leaf RWC was measured according to the method of Yoo et al. (2010). Water loss in the detached leaf was determined according to the method of Whittaker (1994). Leaves were excised from plants, weighed to determine their initial weight, placed with their adaxial surfaces upward on a petri dish in a light incubator (at a temperature of $25 \pm 2{ }^{\circ} \mathrm{C}$ and a relative humidity of $60 \%$ ) and reweighed at $30 \mathrm{~min}$ intervals for up to $6 \mathrm{~h}$ to obtain the actual time fresh weight. The water loss was calculated as follows: (initial weight - actual time fresh weight)/initial weight $\times 100$.

\section{Measurement of Endogenous $\mathrm{H}_{2} \mathrm{~S}$ Concentration}

Endogenous $\mathrm{H}_{2} \mathrm{~S}$ was measured by the formation of methylene blue from dimethyl-p-phenylenediamine in $\mathrm{H}_{2} \mathrm{SO}_{4}$ according to the method described by Sekiya et al. (1982) and Zhang et al. (2009) with some little modifications. Leaves $(0.3 \mathrm{~g})$ were ground and then extracted with $3 \mathrm{ml}$ of phosphate buffer solution ( $\mathrm{pH}$ 6.8, $50 \mathrm{mM}$ ) including $0.2 \mathrm{M}$ ascorbic acid and 0.1 M EDTA. Subsequently, $1 \mathrm{M} \mathrm{HCl}(0.5 \mathrm{ml})$ was added to the homogenate in a test tube for releasing $\mathrm{H}_{2} \mathrm{~S}$, and $0.5 \mathrm{ml}$ of $1 \%(\mathrm{w} / \mathrm{v})$ zinc acetate was used to absorb $\mathrm{H}_{2} \mathrm{~S}$. After 30 min of reaction, $5 \mathrm{mM}$ dimethylp-phenylenediamine $(0.3 \mathrm{ml})$ dissolved in $3.5 \mathrm{mM} \mathrm{H}_{2} \mathrm{SO}_{4}$ was added to the trap. Then, $50 \mathrm{mM}$ ferric ammonium sulfate in $100 \mathrm{mM} \mathrm{H}_{2} \mathrm{SO}_{4}$ was injected into the trap. The amount of $\mathrm{H}_{2} \mathrm{~S}$ in the zinc acetate traps was determined at OD $667 \mathrm{~nm}$ after incubating the mixture for $15 \mathrm{~min}$ at room temperature. Blanks were prepared using the same procedures without the zinc acetate solution.

\section{Gas Exchange and Chlorophyll Fluorescence Quenching Analyses}

The gas exchange of fully expanded leaves (the number leaves of a plant was about 12-14), including transpiration, stomatal conductance, and net $\mathrm{CO}_{2}$ assimilation, was determined using a portable photosynthesis system (Li-6400, Li-Cor, Lincoln, NE, USA). Plants were grown for 6 weeks under short-day conditions $(10 \mathrm{~h}$ light). Such conditions resulted in plants with leaves large enough to fill the $6-\mathrm{cm}^{2} \mathrm{Li}-6400$ chamber. Gas exchange was measured at PAR levels of 1200, 800, 600, 400, 200, 100, 50, 20, 10 , and $0 \mu \mathrm{mol} \mathrm{m}^{-2} \mathrm{~s}^{-1}$. All measurements were conducted in the morning (9:00-11:30) to avoid the high temperature and air vapor pressure deficits in the afternoon. Light was supplemented using an LED light system. Using non-rectangular hyperbola modeling, the response of leaf Pn to PAR was calculated with respect to the apparent dark respiration $(R \mathrm{~d})$, light compensation point $(L c p)$, light saturation point ( $L s p)$, apparent quantum yield $(A Q E)$ and maximal net photosynthetic rate $(P \max )$, as described by Prioul and Chartier (1977).

$$
\begin{aligned}
& \mathrm{Pn}=\mathrm{AQE} \times \mathrm{PAR}+\mathrm{P} \max - \\
& \frac{\sqrt{(A Q E \times P A R+P \max )^{2}-4 A Q E \times \theta \times P A R \times P \max }}{2 \theta}-R d,
\end{aligned}
$$

where $\theta$ is the convexity, quantum efficiency is the initial slope of the curve, and $R \mathrm{~d}$ is the point at which the curve crosses the $y$ axis at PAR $=0$, and the $L \mathrm{cp}$ at which the curve crosses the $x$ axis. The vapor pressure deficit (VPD) during measurement was $\sim 1 \mathrm{kPa}$. The instantaneous water use efficiency (WUE) was calculated as the net $\mathrm{CO}_{2}$ assimilation rate divided by the transpiration rate.

Chlorophyll fluorescence is considered a tool for interpreting the stress tolerance of plants by evaluating the physiological status of the plant and the state of Photosystem II (PSII) (Krause and Weis, 1991; Peeva and Cornic, 2009). The chlorophyll fluorescence of S. oleracea was measured using a Plant Efficiency Analyzer (Hansatech Instruments Ltd., Norfolk, England). The ratio of variable $(\mathrm{Fv})$ to maximum fluorescence $(\mathrm{Fm})$ was 
measured in four seedlings per pot. The leaves were darkadapted for more than $30 \mathrm{~min}$ prior to the measurement. The minimum fluorescence (Fo), the Fm, the variable fluorescence $(\mathrm{Fv}=\mathrm{Fm}-\mathrm{Fo})$ and the ratio of $\mathrm{Fv} / \mathrm{Fm}$ were recorded for $15 \mathrm{~s}$ at a $100 \%$ intensity level of the photon flux density $\left(4000 \mu \mathrm{mol} \mathrm{m} \mathrm{m}^{-2}\right.$ $\left.s^{-1}\right)$. Additionally, the steady-state fluorescence level reached upon continuous illumination $\left(\mathrm{Fs}^{\prime}\right)$, the maximal fluorescence level induced by a saturating light pulse at the steady-state $\left(\mathrm{Fm}^{\prime}\right)$, and the minimum fluorescence level after a $3 \mathrm{~s}$ period of farred light required to oxidize the plastoquinone pool $\left(\mathrm{Fo}^{\prime}\right)$ were measured and used to calculate other parameters as follows: The quantum yield of PSII photochemistry (PSII) was calculated as $1-\left(\mathrm{Fs}^{\prime} / \mathrm{Fm}^{\prime}\right)$. The electronic transport ratio (ETR) was calculated as $\mathrm{PAR} \times \mathrm{PSII} \times 0.85 \times 0.5$. Non-photochemical quenching $(\mathrm{NPQ})$ was calculated as $\left(\mathrm{Fm} / \mathrm{Fm}^{\prime}\right)-1$. Photochemical quenching (qP) was calculated as $\left(\mathrm{Fm}^{\prime}-\mathrm{Fs}\right) /\left(\mathrm{Fm}^{\prime}-\mathrm{Fo}^{\prime}\right)$ (Krause and Weis, 1991).

\section{Measurement of Leaf Water Potential and Osmotic Potential}

Leaf water potential was measured after plants were subjected to drought for 0,5 and 8 days and re-watered for 1 and 4 days. The fully expanded leaves were selected and measured between 10:00 and 11:00 A.M. using a pressure chamber (Model 1000, PMS Instrument Co., Corvallis, OR, USA). The osmotic potential was measured using a dew point microvolt meter (Model 5520, Wescor, Logan, UT, USA). Leaf water and osmotic potentials were calculated according to the method of Yin et al. (2013). Each treatment included six replicates.

\section{Measurement of Superoxide Radical, Hydrogen Peroxide and Lipid Peroxidation}

The production of superoxide radical $\left(\mathrm{O}_{2}^{-\bullet}\right)$ was measured using the method of Wang and Luo (1990) with some modifications. Hydrogen peroxide $\left(\mathrm{H}_{2} \mathrm{O}_{2}\right)$ was measured spectrophotometrically according to Alexieva et al. (2001). The lipid peroxidation level was determined in terms of malondialdehyde (MDA) content via the thiobarbituric acid (TBA) reaction as described by Dhindsa et al. (1981).

\section{Measurement of Osmolyte Content}

Proline content was determined according to the method of Bates et al. (1973). Glycinebetaine content was measured using an HPLC Shimadzu-V analytical procedure according to the method of Bessieres et al. (1999) with some modifications. Frozen leaf samples in liquid nitrogen were homogenized with $4 \mathrm{ml}$ water: chloroform: methanol (3: 5: $12 \mathrm{v} / \mathrm{v} / \mathrm{v}$ ) solution and incubated overnight at $4^{\circ} \mathrm{C}$. The upper methanolic phase $(1 \mathrm{ml})$ of the extract was purified using BioRad AG1-X8 ion exchange resin. The ion exchange resin was removed by centrifugation at $5000 \mathrm{~g}$ for $10 \mathrm{~min}$, and the supernatant was filtered using a $0.22 \mu \mathrm{m}$ membrane filter before being loaded into the HPLC system. A Nucleogel RP column (RP-S 100-8, $300 \times 7.7 \mathrm{~mm}$ ) proceeded by a guard column was used, and the mobile phase $(15 \mathrm{mM}$ $\mathrm{KH}_{2} \mathrm{PO}_{4}$ ) was delivered by an analytical isocratic pump at a flow rate of $0.7 \mathrm{ml} \mathrm{min}^{-1}$ at $70^{\circ} \mathrm{C}$. The $\mathrm{GB}$ content was measured using a UV detector at $192 \mathrm{~nm}$, and quantification was performed by comparing the peak surface areas.

\section{Measurement of Glucose, Fructose, Sucrose, and Trehalose Content}

Glucose and sucrose were measured according to the method of Zhao et al. (2004) using the Shimadzu sugar analysis system (HPLC, Shimadzu, Kyoto, Japan). Leaves were homogenized in $7 \mathrm{ml} \mathrm{85 \%} \mathrm{(v/v)} \mathrm{ethanol,} \mathrm{the} \mathrm{first} 5 \mathrm{ml}$ aliquot was adjusted to $1 \mathrm{mM}$ with respect to lactose and used as an internal standard. The slurry was transferred to a $10 \mathrm{ml}$ tube, and the mortar was washed with an additional $2 \mathrm{ml} \mathrm{80 \%} \mathrm{ethanol,} \mathrm{which} \mathrm{was} \mathrm{added}$ to the tube. The suspensions were heated at $80^{\circ} \mathrm{C}$ for $30 \mathrm{~min}$ and centrifuged at $12000 \mathrm{~g}$ for $20 \mathrm{~min}$, and then the supernatant was collected. The supernatant was heated at $95^{\circ} \mathrm{C}$ for $2 \mathrm{~h}$ to remove ethanol, and the final sample $(\sim 500 \mu \mathrm{l})$ was frozen at $-80^{\circ} \mathrm{C}$ for $24 \mathrm{~h}$. Subsequently, the sample was lyophilized to dryness, reconstituted in $500 \mu \mathrm{l}$ distilled, deionized water, and centrifuged at $12000 \mathrm{~g}$ for $20 \mathrm{~min}$. Then, the supernatant was passed through a membrane filter (pore size $0.22 \mu \mathrm{m}$ ) for analysis. Sugars in the extracts were injected into an eluent of $19 \mathrm{mM} \mathrm{NaOH}$ delivered at $1 \mathrm{ml} \mathrm{min}{ }^{-1}$ using a Waters 1525 HPLC pump (Waters 1525/VIS 2424 Detector, Breeze ${ }^{\mathrm{TM}}$, USA) and separated by anion exchange using an XBridge ${ }^{\mathrm{TM}}$ amide C18 column $(4.6 \times 250 \mathrm{~mm}, 3.5 \mu \mathrm{m}$ particle size) with guard column. Each treatment included four replicates.

Fructose and trehalose contents were estimated using fructose and trehalose reagent kits, respectively (Shanghai Solarbio Biocompany, Shanghai, China). The absorbances of fructose and trehalose were measured at 530 and $520 \mathrm{~nm}$, respectively, following the manufacturer's instructions.

\section{Measurement of Polyamines Content}

Polyamines (PAs) were extracted according to the method of Flores and Galston (1982) and Duan et al. (2008). Briefly, leaves were ground in $1 \mathrm{ml}$ of $5 \%(\mathrm{v} / \mathrm{v})$ cold perchloric acid and then incubated at $4^{\circ} \mathrm{C}$ for $1 \mathrm{~h}$. After incubation, the homogenate was centrifuged at $12000 \mathrm{~g}$ for $30 \mathrm{~min}$ at $4^{\circ} \mathrm{C}$. To measure the conjugated PAs, the plant extract residues were washed with $5 \%$ $\mathrm{HClO}_{4}$ and then subjected to hydrolysis in $6 \mathrm{M} \mathrm{HCl}$ at $110^{\circ} \mathrm{C}$ for $16 \mathrm{~h}$. The filtered hydrolysates were allowed to evaporate to dryness, and the residues were dissolved in $5 \% \mathrm{HClO}_{4}$ for the measurement of conjugated PAs. Subsequently, the supernatant and the suspension residues were benzoylated as follows: A $500 \mu \mathrm{l}$ volume of supernatant was treated with $2 \mathrm{ml}$ of $2 \mathrm{~N} \mathrm{NaOH}$ and $10 \mu \mathrm{l}$ benzoyl chloride, and the mixture was then vortexed for $30 \mathrm{~s}$ and incubated for $30 \mathrm{~min}$ at $37^{\circ} \mathrm{C}$. The reaction was terminated by adding $2 \mathrm{ml}$ saturated $\mathrm{NaCl}$ solution. Next, the benzoyl polyamine was extracted with $2 \mathrm{ml}$ cold diethyl. Ultimately, $1 \mathrm{ml}$ ether phase was evaporated to dryness and dissolved in $1 \mathrm{ml}$ of methanol for the determination of endogenous polyamines. Eventually, high performance liquid chromatography (Waters 1525/UVD 2489 Detector, Breeze ${ }^{\text {TM }}$, USA) was used to analyze the endogenous PA content. Briefly, $20 \mu \mathrm{l}$ of benzoyl PAs were injected into the loop and then loaded onto a Waters Symmetry C18 column $(7.5 \mathrm{~cm} \times 4.6 \mathrm{~mm}, 3.5 \mu \mathrm{m}$ particle size $)$. The column 
A

\section{Relative soil Control $100 \mu \mathrm{M} \mathrm{NaHS}$ water content}

(\%)

100

50
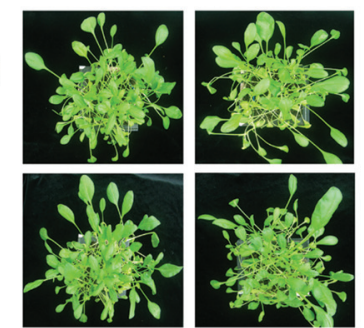

29
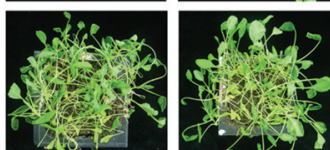

10

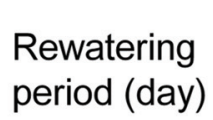

(1)
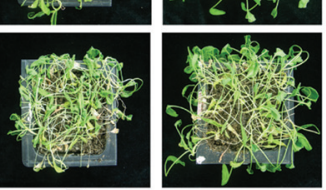

Recovery
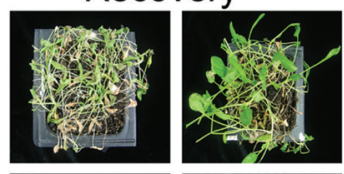

4

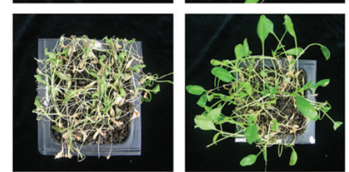

B

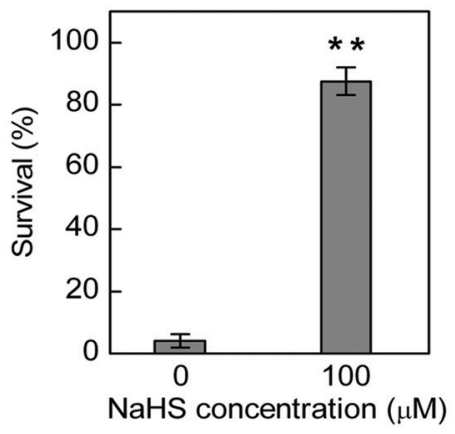

C

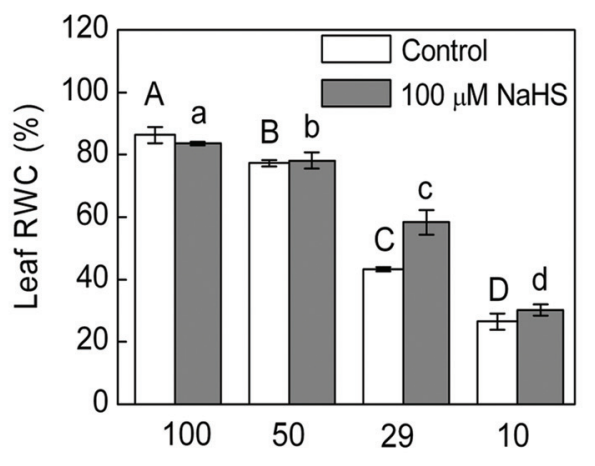

Relative soil water content (\%)

FIGURE 1 | Plant water stress response was analyzed in 6-week-old NaHS-treated Spinacia oleracea seedlings grown under drought and rewatering conditions. Four containers of each treatments were determined in three independent experiments. Relative soil water content is the soil water relative to the soil water at day 0 of withholding water and is the average of four containers. The photograph in (A) illustrates a representative result of one replicate from one experiment. Four days after rewatering, plant survival was determined (mean $\pm \mathrm{SE}, n=4)(\mathbf{B})$. In a separate experiment, 6 -week-old seedlings were exposed to water deficit stress by withholding water. Leaf RWC (mean $\pm \mathrm{SE}, n=6$ ) was determined (C). In (B), the significant level of the difference between control and treatment is indicated by two asterisks ${ }^{\star \star} P<0.01$. In (C), the columns labeled with different letters indicate significant differences at $P<0.05$.

temperature was maintained at $30^{\circ} \mathrm{C}$ for PAs. The mobile phase was methanol- $\mathrm{H}_{2} \mathrm{O}(59: 41, \mathrm{v}: \mathrm{v})$. The PA peaks were detected with a UV detector at $230 \mathrm{~nm}$ at a flow rate of $0.7 \mathrm{ml} \mathrm{min}^{-1}$.

\section{Total RNA Extraction and Gene Expression Analysis}

Frozen leaves $(0.5 \mathrm{~g})$ were ground in liquid nitrogen with $2 \%$ polyvinylpyrrolidone and extracted with an RNA purification reagent (Invitrogen Inc., Carlsbad, CA, USA) according to the manufacturer's instructions. The RNA content was determined with an ultraviolet spectrophotometer (Cary 50, Varian, Agilent), and the integrity of the RNA was evaluated by $1 \%$ agarose gel electrophoresis. Total RNA was reverse-transcribed into firststrand cDNAs using M-MLV reverse transcriptase (TaKaRa, Dalian, China). A 10- $\mu$ l real-time PCR reaction contained the following: $0.6 \mu \mathrm{l}$ of forward and reverse primers for the sugar biosynthesis-related genes, PA biosynthesis-related genes, betaine aldehyde dehydrogenase $(S o B A D H)$, choline monooxygenase
(SoCMO), and aquaporin (SoPIP1;2) (listed in Supplementary Table S1); $1 \mu \mathrm{l}$ of cDNA (equivalent to $10 \mathrm{ng}$ of mRNA) and $5 \mu \mathrm{l}$ of FastStart Universal SYBR Green Master Mix (ROX, Mannheim, Germany). The amplification and detection of the dsDNA synthesis of these genes were performed using the PCR conditions described in Supplementary Table S1. Three independent replicates were performed for each sample. The comparative threshold cycle $\left(\mathrm{C}_{\mathrm{t}}\right)$ method was used to determine the relative amount of gene expression. The glyceraldehyde3-phosphate dehydrogenase gene (SoGADPH) was used as an internal control. The mRNA transcriptional abundance value of the genes was expressed as $2^{-\Delta \Delta \mathrm{Ct}}$ (Livak and Schmittgen, 2001). A LightCycler 480II Real-Time PCR System (Roche, Bern, Switzerland) was used to perform qRT-PCR.

\section{Statistical Analysis}

At least six leaves were used to measure gas exchange and chlorophyll fluorescence quenching. At least three replicates were conducted for physiological and biochemical analyses. 


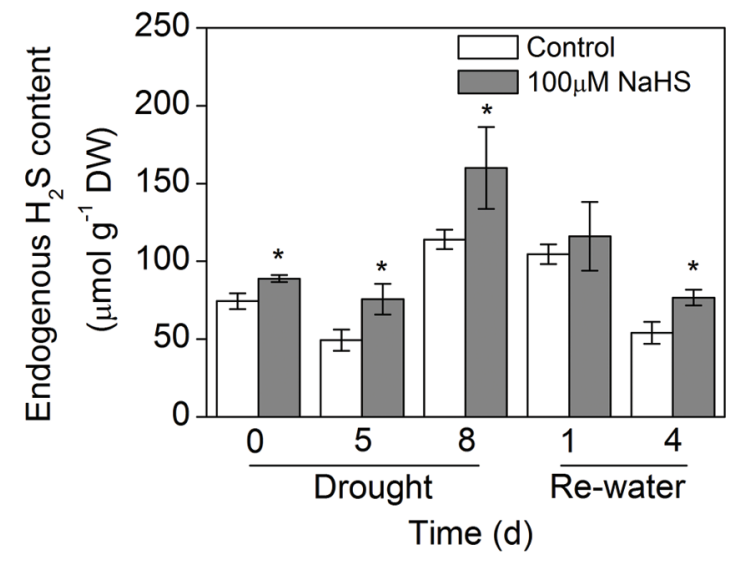

FIGURE 2 | Effect of $\mathrm{H}_{2} \mathrm{~S}$ donor NaHS on the endogenous $\mathrm{H}_{2} \mathrm{~S}$ concentration in 6-week-old $S$. oleracea seedlings leaves after drought for $\mathbf{0 , 5}$, and $\mathbf{8}$ days and rewatering for $\mathbf{1}$ and $\mathbf{4}$ days. The significant level of the difference between control and treatment is indicated by an asterisk $* P<0.05$.

One-way or two-way ANOVA procedure with the SPSS 19.0 software (SPSS Inc., Chicago, IL, USA) were used for analyzing statistical significances, and the results are expressed as the mean values \pm SE. Post hoc comparisons were tested using the Tukey test at a significance level of $P<0.05$.

\section{RESULTS}

\section{Effect of $\mathrm{H}_{2} \mathrm{~S}$ Donor on Plant Water Stress Responses}

Under drought conditions, and thus under low relative SWC, S. oleracea seedlings treated with $100 \mu \mathrm{M}$ NaHS, a donor of $\mathrm{H}_{2} \mathrm{~S}$, survived better than untreated control plants (Figure 1A). Most of the control plants wilted at $10 \pm 1.5 \%$ SWC, while most of the NaHS-treated plants presented less severe leaf wilting symptoms (Figure 1A). Additionally, on the first day after rewatering, the NaHS-treated seedlings recover more quickly than the control plants (Figure 1A). Moreover, more than $80 \%$ of the NaHS-treated plants and $<10 \%$ of the control plants survived to the fourth day after re-watering (Figure 1B). Increased drought survival of the NaHS-treated plants was associated with a higher capacity to maintain RWC than the control plants at $29 \pm 2.5 \%$ SWC (Figure 1C). To understand the physiological mechanism through which the NaHS-treated plants acquired water stress tolerance by maintaining higher leaf water content under low soil moisture conditions, we analyzed the effect of different concentrations of NaHS on the RWC of detached leaves of $S$. oleracea seedlings. The RWC of detached $S$. oleracea seedling leaves treated with various concentrations of NaHS (10$1000 \mu \mathrm{M})$ were significantly higher than that of control plants, with an optimal NaHS concentration of $100 \mu \mathrm{M}$ (Supplementary Figure S1A). Indeed, the detached leaves treated with $100 \mu \mathrm{M}$ NaHS exhibited the highest leaf RWC and the lowest leaf water loss ratio of all plants (Supplementary Figures S1B,C). The leaf water loss of NaHS-treated plants decreased by $~ 10 \%$ compared with that of the control plants (Supplementary Figure S1D).

\section{Effect of $\mathrm{H}_{2} \mathrm{~S}$ Donor on the Endogenous $\mathrm{H}_{2} \mathrm{~S}$ Concentration}

To determine whether exogenous $\mathrm{H}_{2} \mathrm{~S}$ affects endogenous $\mathrm{H}_{2} \mathrm{~S}$ concentrations in $S$. oleracea seedlings suffering from drought stress, we measured the endogenous $\mathrm{H}_{2} \mathrm{~S}$ concentration of $S$. oleracea seedling leaves. As shown in Figure 2, 8 days of drought stress promoted endogenous $\mathrm{H}_{2} \mathrm{~S}$ production in S. oleracea seedlings. Moreover, exogenously applied NaHS also increased the production of endogenous $\mathrm{H}_{2} \mathrm{~S}$ under drought stress. However, re-watering for 4 days inhibited the droughtinduced endogenous production of $\mathrm{H}_{2} \mathrm{~S}$ in $\mathrm{S}$. oleracea seedling leaves (Figure 2).

\section{Effect of the $\mathrm{H}_{2} \mathrm{~S}$ Donor on Leaf Photosynthesis and Electron Transport Efficiency of PSII}

There is a close relationship between water stress and photosynthesis in plants. Accordingly, we measured the photosynthesis of $S$. oleracea seedlings treated with $\mathrm{NaHS}$ under drought condition. The net $\mathrm{CO}_{2}$ assimilation rate of the NaHS-treated plants was significantly increased compared with that of control plants (Figure 3A). Additionally, the stomatal conductance of the NaHS-treated plants was somewhat higher than that of the control plants, although the difference was not statistically significant (Figure 3B). The internal $\mathrm{CO}_{2}$ concentration in the leaves of NaHS-treated plants was higher than in those of the control plants (Figure 3C). Additionally, at saturating light levels, the leaf transpiration rate of the NaHS-treated plants was higher than that of the control plants (Figure 3D). However, the instantaneous WUE values of the NaHS-treated plants and the control plants were similar (Figure 3E). Additionally, the VPD of the NaHS-treated plants was higher than that of the control plants (Figure 3F). Moreover, the $R \mathrm{~d}, L \mathrm{cp}, L \mathrm{sp}, A Q E$, and $P \max$ of $S$. oleracea plants were calculated by modeling the response of the leaf Pn to PAR via a non-rectangular hyperbola (Table 1). Lcp, Lsp, and Pmax were notably higher in the NaHS-treated plants than in the controls. These results demonstrate that NaHS treatment increased the photosynthesis of $S$. oleracea seedlings under drought condition.

Chlorophyll fluorescence is considered a tool for interpreting stress tolerance in plants. Therefore, we measured this key trait in $S$. oleracea seedlings under drought stress. Parameters indicative of chlorophyll fluorescence (PSII, ETR, Fv/Fm, Fv'/Fm' and qP) were significantly decreased in the NaHS-treated and the control plants when the seedlings were under drought condition for 5 and 8 days compared with the water sufficient plants (0 day) (Figures 4A-D,F). Interestingly, PSII and $\mathrm{Fv}^{\prime} / \mathrm{Fm}^{\prime}$ in the NaHStreated plants were higher than in the control plants on the first and fourth day after re-watering (Figures 4A,D). The ETR of the NaHS-treated plants was obviously increased compared with that of control plant throughout the entire treatment period, including 8 days of drought and 4 days of re-watering (Figure 4B). In contrast, NPQ in the NaHS-treated plants was 

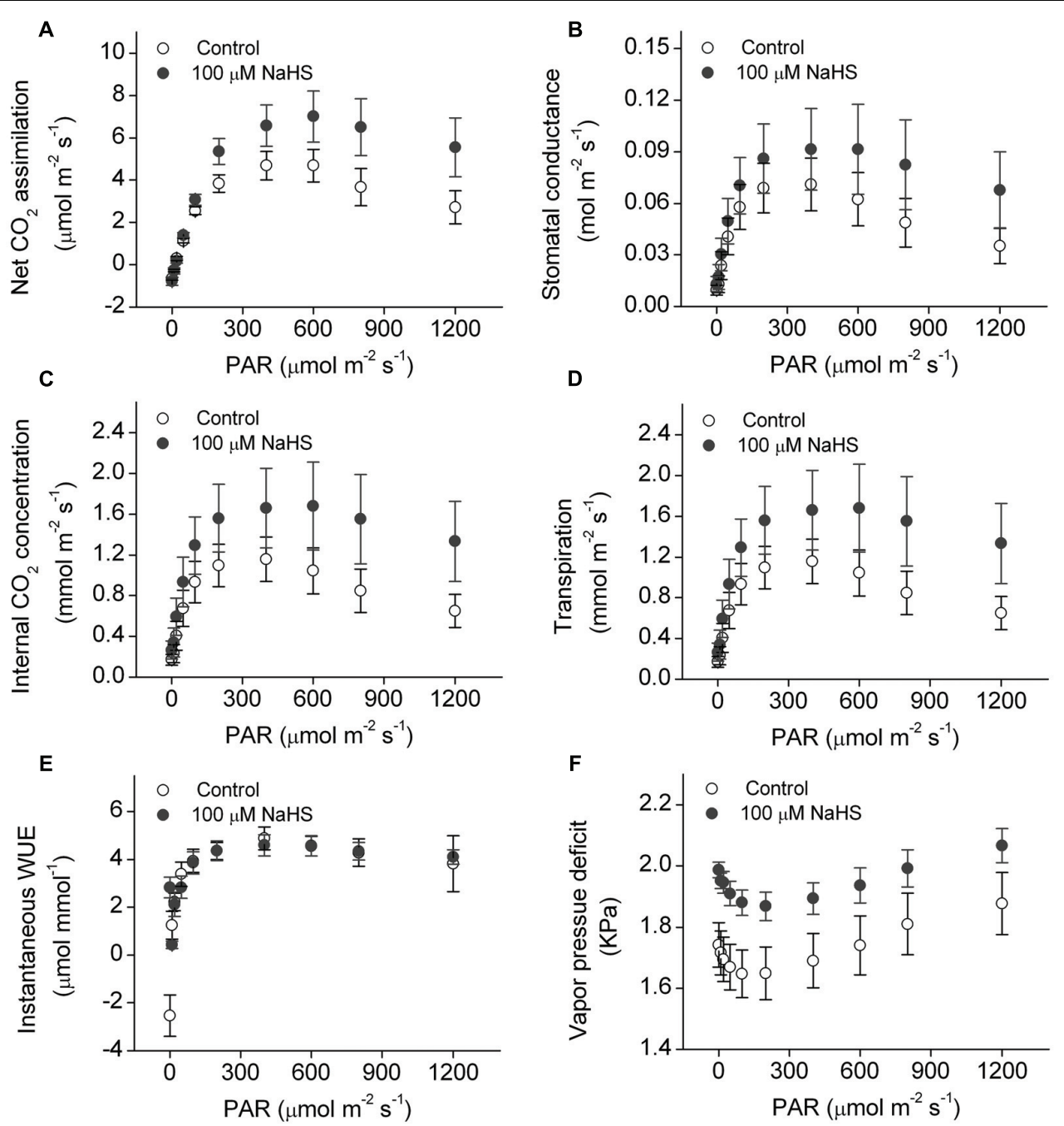

FIGURE 3 | Effect of $\mathrm{H}_{2} \mathrm{~S}$ donor NaHS on net $\mathrm{CO}_{2}$ assimilation (A), stomatal conductance (B), internal $\mathrm{CO}_{2}$ concentration (C), transpiration (D), instantaneous WUE (E), and vapor pressure deficit (F) of 6-week-old S. oleracea seedlings leaves under drought condition. Each value represents the mean $\pm \operatorname{SE}(n=6)$.

significantly decreased compared with that of control plants throughout the entire treatment period (Figure $4 \mathrm{E}$ ). The above results show that compared with the control plants, the NaHStreated $S$. oleracea plants exhibited the high electron transport efficiency under drought condition.

\section{Effect of $\mathrm{H}_{2} \mathrm{~S}$ Donor on Leaf Water Potential and Osmotic Potential}

The leaf water potential of the NaHS-treated plants and the control plants grown under drought condition underwent a significant decrease, especially on the eighth day after drought, when the NaHS-treated plant exhibited a higher leaf water potential than the control plants. After re-watering, the leaf water potential experienced a sharp increase in the NaHS-treated and control plants, and the NaHS-treated plants again showed a higher leaf water potential than the control plants (Figure 5A). However, the leaf osmotic potential of the NaHS-treated plants
TABLE 1 | Effects of $\mathrm{H}_{2} \mathrm{~S}$ on apparent quantum yield (AQE), dark respiration $(R d)$, low light compensation point (Lcp), high light saturation point (Lsp), and maximum net photosynthetic rate (Pmax) of S. oleracea seedlings under drought condition.

\begin{tabular}{lcc}
\hline Variables & Control & $\mathbf{1 0 0} \boldsymbol{\mu}$ M NaHS \\
\hline$A Q E$ & $0.050 \pm 0.005$ & $0.051 \pm 0.003$ \\
$R \mathrm{~d}\left(\mu \mathrm{mol} \mathrm{CO} \mathrm{m}^{-2} \mathrm{~s}^{-1}\right)$ & $0.814 \pm 0.096$ & $0.890 \pm 0.093$ \\
$\operatorname{Lcp}\left(\mu \mathrm{mol} \mathrm{m} \mathrm{s}^{-1}\right)$ & $16.11 \pm 1.00$ & $19.30 \pm 0.80^{*}$ \\
$\operatorname{Lsp}\left(\mu \mathrm{mol} \mathrm{m} \mathrm{m}^{-2} \mathrm{~s}^{-1}\right)$ & $236.9 \pm 39.85$ & $382.9 \pm 24.01^{* *}$ \\
$P \max \left(\mu \mathrm{mol} \mathrm{CO} \mathrm{m}^{-2} \mathrm{~s}^{-1}\right)$ & $4.92 \pm 0.71$ & $7.64 \pm 1.01^{*}$
\end{tabular}

Data are presented as mean $\pm S E$. The significant level of difference between control and treatment is indicated by asterisk $* P<0.05$ and ${ }^{* *} P<0.01$.

and the control plants showed no significant difference under drought and re-watering conditions except for the drought at 0 and 8 days (Figure 5B). 
A

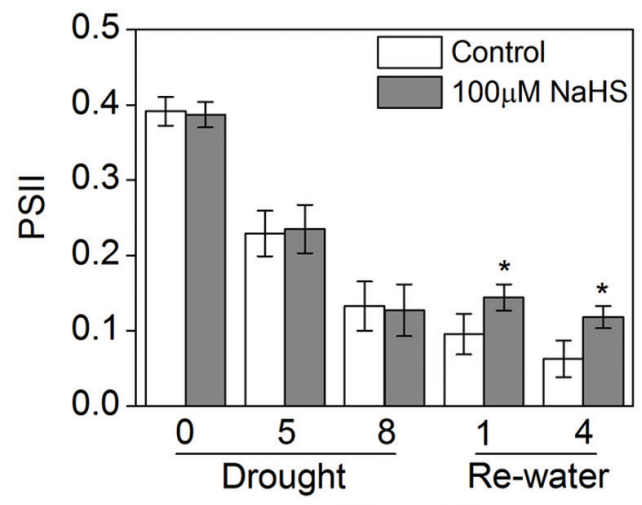

C

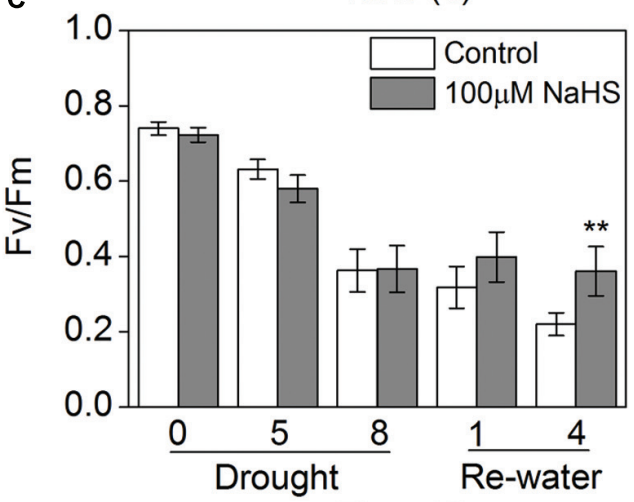

E

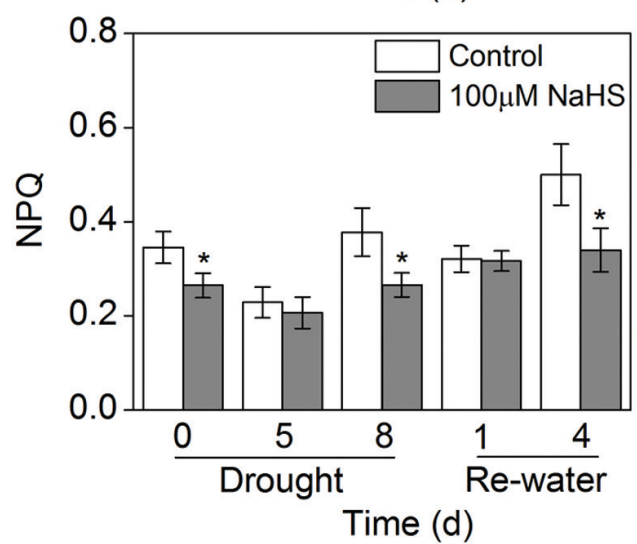

B

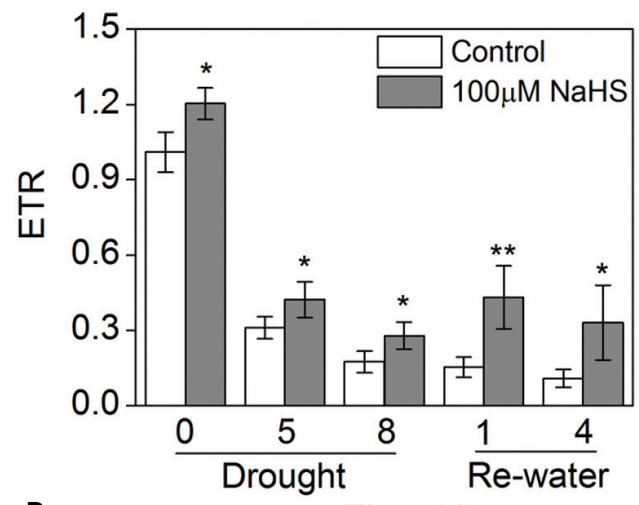

Time (d)

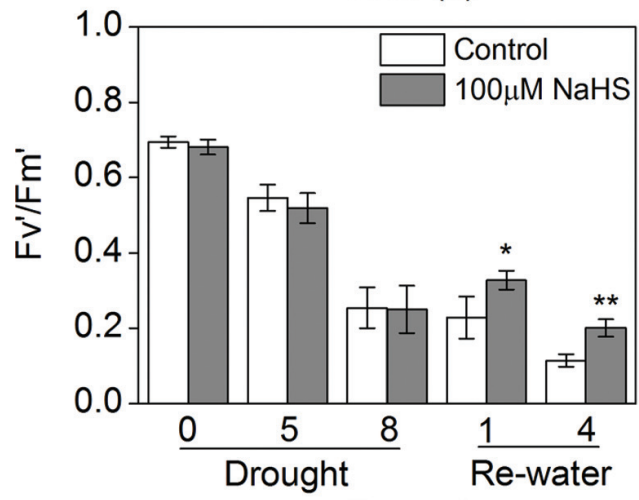

$\mathbf{F}$

Time (d)

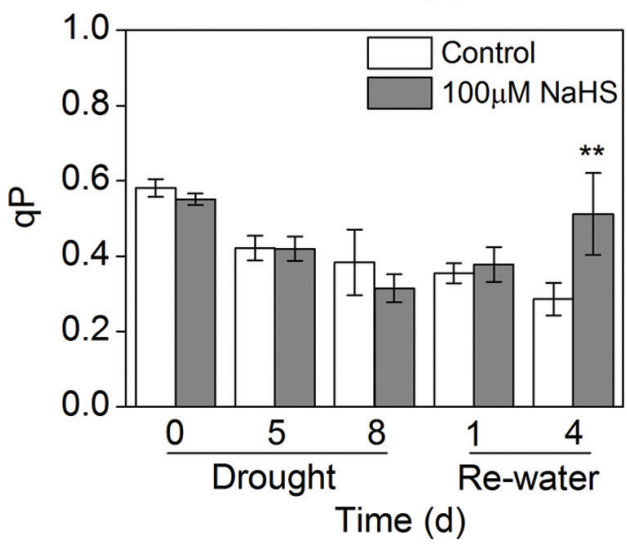

FIGURE 4 | Effect of $\mathrm{H}_{2} \mathrm{~S}$ donor NaHS on PSII (A), ETR (B), Fv/Fm (C), Fv'/Fm' (D), NPQ (E) and qP (F) in 6-week-old S. oleracea seedlings leaves after drought for $\mathbf{0 , 5}$, and $\mathbf{8}$ days and rewatering for $\mathbf{1}$ and $\mathbf{4}$ days. The significant level of the difference between control and treatment is indicated by an asterisk $* P<0.05$ and ${ }^{* *} P<0.01$.

\section{Effect of $\mathrm{H}_{2} \mathrm{~S}$ Donor on Superoxide Radical Production, Hydrogen Peroxide Production, and Lipid Peroxidation Content}

To further confirm the protective role of $\mathrm{H}_{2} \mathrm{~S}$ in the plant response to drought stress, some simple physiological parameters were measured in $S$. oleracea seedlings. The production rate of superoxide radical $\left(\mathrm{O}_{2}^{-\bullet}\right)$ had no obvious difference between the NaHS-treated plants and the control plants under drought condition. However, on the first day after re-watering, the $\mathrm{O}_{2}^{-\bullet}$ content significantly decreased in the NaHS-treated plants (Figure 6A). Additionally, there was a significant decrease in the accumulation of $\mathrm{H}_{2} \mathrm{O}_{2}$ in NaHS-treated plants compared with the control plants throughout the treatment period, except for 4 days after re-watering (Figure 6B). Similarly, under drought condition, the content of lipid peroxidation (MDA) underwent a sharp decrease in the NaHS-treated plants 

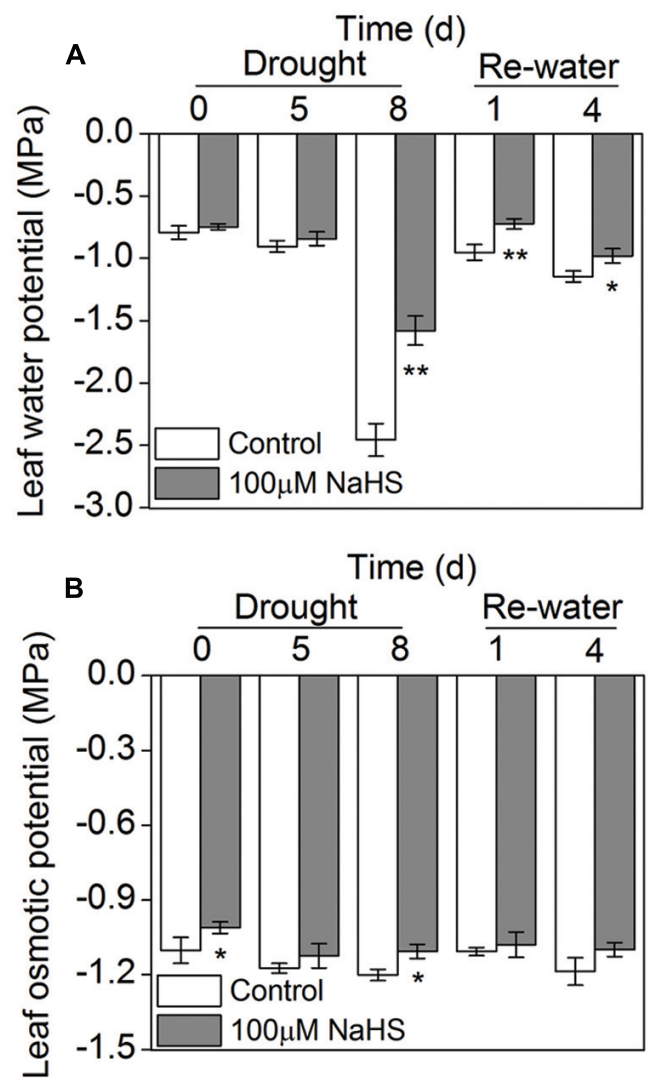

FIGURE 5 | Effect of $\mathrm{H}_{2} \mathrm{~S}$ donor NaHS on leaf water potential (A) and leaf osmotic potential (B) in 6-week-old $S$. oleracea seedlings leaves after drought for $\mathbf{0 , 5}$, and $\mathbf{8}$ days and rewatering for $\mathbf{1}$ and $\mathbf{4}$ days. The significant level of the difference between control and treatment is indicated by an asterisk ${ }^{*} P<0.05$ and ${ }^{* *} P<0.01$

compared with the control plants, but the MDA content was not significantly different between treatments after re-watering (Figure 6C). These results show that $\mathrm{H}_{2} \mathrm{~S}$ can protect $S$. oleracea seedlings against drought through the inhibition of oxidative stress.

\section{Effect of $\mathrm{H}_{2} \mathrm{~S}$ Donor on Proline and GB Content}

NaHS treatment had no obvious effect on the proline content under drought condition, but on the fourth day after rewatering the proline content had an obvious decrease in the NaHS-treated plants (Figure 6D). Furthermore, GB content in NaHS-treated plants had obvious increase under drought stress for 5 and 8 days compared with the control plants, respectively (Figure 6E). However, NaHS had no significant effect on the GB content under re-watering for 1 and 4 days compared with the control plants, respectively (Figure 6E). Besides, we also found that drought stress for 8 days could increase the GB content of $S$. oleracea seedlings leaves compared with the control plants (0 day) (Figure 6E).

\section{Effect of $\mathrm{H}_{2} \mathrm{~S}$ Donor on Sugar Biosynthesis}

The glucose content in the NaHS-treated plants decreased to varying degrees compared with the control plants under drought and re-watering conditions (Figure 7A). In contrast, the fructose content in the NaHS-treated plants increased considerably compared with that of control plants under the same drought and re-watering conditions (Figure 7B). The sucrose content in the NaHS-treated plants decreased significantly compared with that of control plants under drought condition, but the content of this sugar increased notably on the first day of re-watering (Figure 7C). Similarly, the trehalose content in the NaHS-treated plants experienced a significant decrease compared with the control plants under drought condition, but after re-watering the trehalose content sharply increased in the NaHS-treated plants (Figure 7D).

\section{Effect of $\mathrm{H}_{2} \mathrm{~S}$ Donor on Polyamine Biosynthesis}

The concentration of total free and conjugated polyamines (PAs) was increased in leaves of the NaHS-treated plants compared with that of control plants under similar drought and rewatering conditions (Figure 8D; Supplementary Figure S2D). In particular, this difference was most evident on the fourth day of re-watering (Figure 8D). Moreover, the free Put content was significantly increased in the NaHS-treated plants after 5 days of drought and 1 day of re-watering (Figure 8A). Similarly, the free Spd content increased substantially in the NaHS-treated plants after 5 days of drought and 1 day of rewatering (Figure 8B). In contrast, NaHS treatment significantly decreased the free Spm content after 5 days of drought, but on the fourth day of re-watering, the Spm content increased notably (Figure 8C). Moreover, the conjugated Put content was significantly increased in the NaHS-treated plants after 1 and 4 days of re-watering (Supplementary Figure S2A). Similarly, the conjugated Spd content increased notably in the NaHS-treated plants under drought and re-watering conditions (Supplementary Figure S2B). Additionally, NaHS treatment altered the conjugated Spm content under drought and rewatering conditions (Supplementary Figure S2C).

\section{Effect of $\mathrm{H}_{2} \mathrm{~S}$ Donor on Transcriptional Expression of Sugar Biosynthesis-Related Genes}

To further study the molecular mechanism of $\mathrm{H}_{2} \mathrm{~S}$-alleviated drought stress in plants, we examined the expression levels of the sugar biosynthesis-related genes, GB biosynthesis-related genes such as SoBADH, SoCMO, and SoPIP1;2 using RT-qPCR analysis in S. oleracea seedlings. The expression level of sucrose phosphate synthase (SoSPS1) in the NaHS-treated plants was significantly increased compared to that of control plants after 0 day of drought and 1 and 4 days of re-watering (Figure 9A). Additionally, the expression level of fructose-1,6-bisphosphatase (SoFBPase) increased to varying degrees in the NaHS-treated 
A

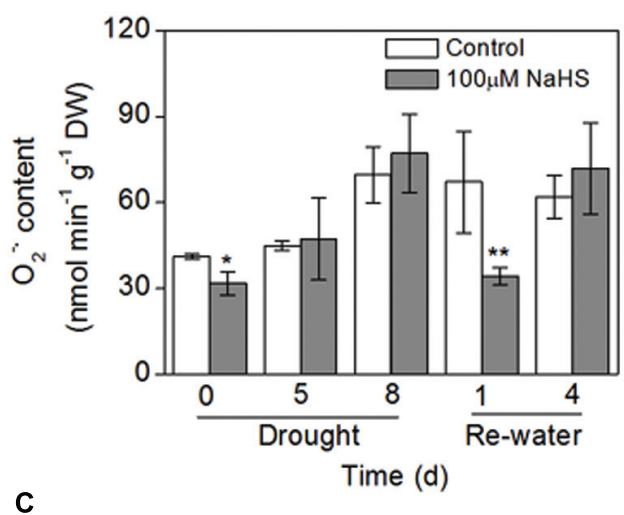

C

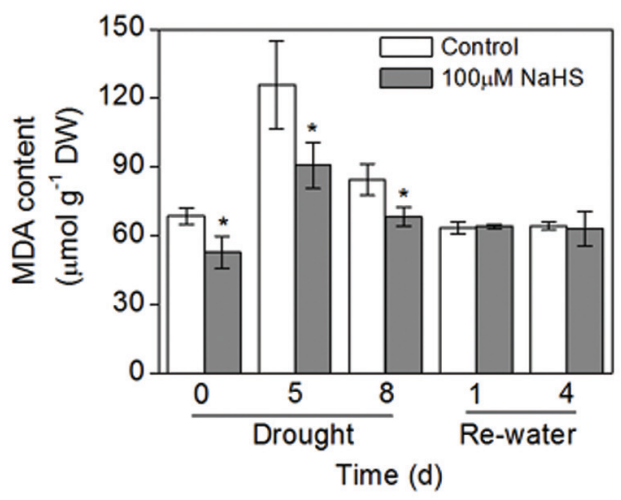

B

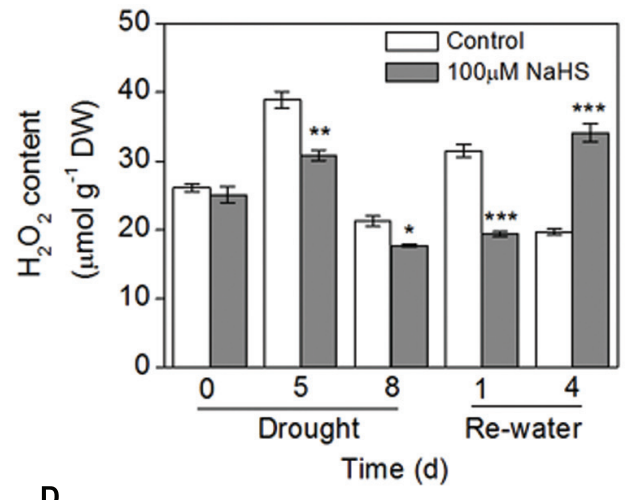

D

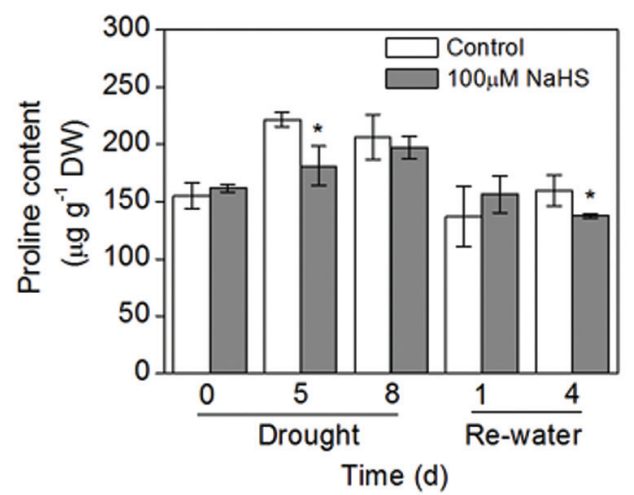

E

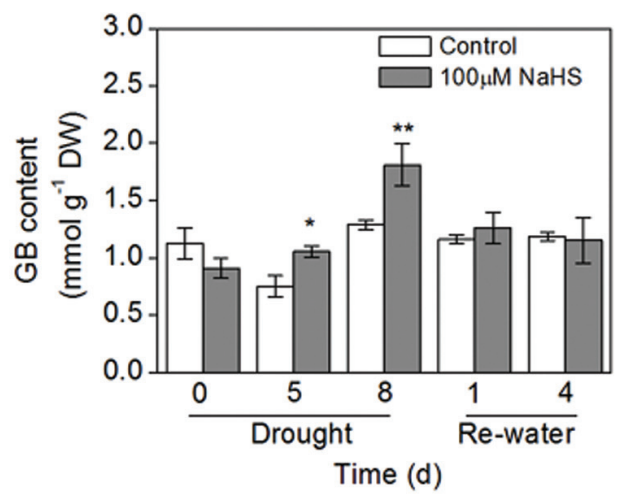

FIGURE 6 | Effect of $\mathrm{H}_{2} \mathrm{~S}$ donor NaHS on the production rate of $\mathrm{O}_{2}^{-\bullet}(\mathrm{A}), \mathrm{H}_{2} \mathrm{O}_{2}$ content (B), MDA content (C), proline content (D) and glycinebetaine (GB) content (E) in 6-week-old $S$. oleracea seedlings leaves after drought for 0,5 , and 8 days and rewatering for $\mathbf{1}$ and 4 days. The significant level of the difference between control and treatment is indicated by an asterisk $* P<0.05,{ }^{* *} P<0.01$, and ${ }^{* * *} P<0.001$.

plants (Figure 9B). Moreover, the expression level of trehalose6-phosphate synthase (SoT6PS) increased clearly in the NaHStreated plants after 5 days of drought, but significantly decreased after 1 day of re-watering (Figure 9C). Furthermore, the expression level of SoBADH in the NaHS-treated plants increased significantly compared with that of control plants after 8 days of drought and 1 and 4 day of re-watering, but the expression level of SoBADH decreased sharply in the NaHS-treated plants after 5 days of drought (Figure 9D). Additionally, the expression abundance of SoCMO in the NaHS-treated plants was evidently enhanced compared with that in control plants after 8 days of drought and 4 days of re-watering, but no significant difference were detected during other treatment periods (Figure 9E). Interestingly, the expression level of SoPIP1;2 in the NaHStreated plants was significantly increased compared with that in the control plants after 5 and 8 days of drought, and an 
A
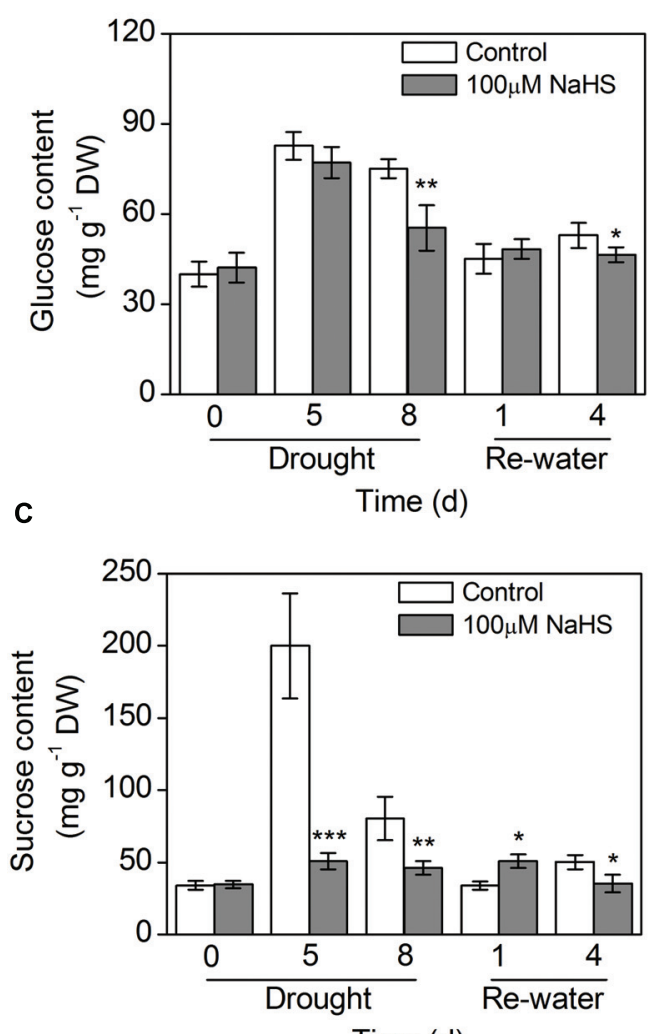

B
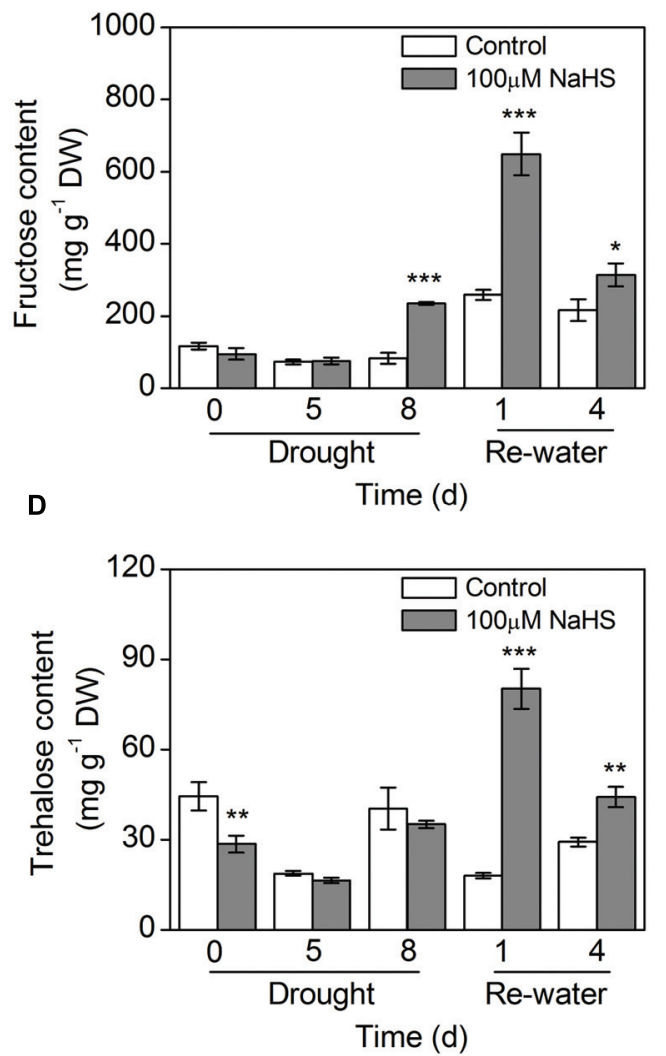

FIGURE 7 | Effect of $\mathrm{H}_{2} \mathrm{~S}$ donor NaHS on glucose content (A), fructose content (B), sucrose content (C), and trehalose content (D) of 6-week-old S. oleracea seedlings leaves after drought for $\mathbf{0 , 5}$, and $\mathbf{8} \mathbf{d}$, and rewatering for $\mathbf{1}$ and $\mathbf{4}$ days. The significant level of the difference between control and treatment is indicated by an asterisk ${ }^{*} P<0.05,{ }^{* *} P<0.01$, and ${ }^{* * *} P<0.001$.

analogous result was also observed after 1 and 4 days of rewatering (Figure 9F).

\section{Effect of $\mathrm{H}_{2} \mathrm{~S}$ Donor on Transcriptional Expression of Polyamines Biosynthesis-Related Genes}

We examined the expression levels of 5 genes involved in PA biosynthesis in $S$. oleracea leaves: arginine decarboxylase (SoADC), N-carbamoylputrescine amidohydrolase (SoCPA), ornithine decarboxylase (SoODC), S-adenosyl-Metdecarboxylase (SoSAMD), and spermidine synthase (SoSPDS) using RT-qPCR analysis. The expression level of SoADC significantly increased in the NaHS-treated plants compared with the control plants after 0,5 , and 8 days of drought, and a comparable result was also observed after 4 days of re-watering (Figure 10A). Moreover, NaHS treatment significantly increased the expression level of SoCPA throughout the experiment in S. oleracea seedlings (Figure 10B). Similarly, NaHS treatment also clearly increased the expression level of SoODC after 0 and 8 days of drought and 4 days of re-watering in $S$. oleracea seedlings (Figure 10C). On the contrary, the expression level of SoSAMD had a significant decrease in the NaHS-treated plants after 0 and 5 days of drought, but no significant difference was observed at other treatment periods (Figure 10D). Furthermore, the expression level of SoSPDS in the NaHS-treated S. oleracea seedlings increased to varying degrees compared with that of the control plants, particularly after 8 days of drought (Figure 10E).

\section{DISCUSSION}

\section{$\mathrm{H}_{2} \mathrm{~S}$ Attenuates Drought Stress-Induced Water Scarcity}

Plants have evolved a variety of strategies to resist or avoid drought stress and reduce the damage caused by lack of water. These strategies include regulating stomatal closure and reducing transpiration, inhibiting the growth rates of leaves or stems, maintaining or increasing the hydraulic conductance of roots and shoots, synthesizing osmotic solutes to maintain cell turgor, and producing antioxidant proteins to retard chlorophyll decomposition (Wilkinson and Davies, 2010). In the present study, under drought stress condition, the survival ratio of plants, the leaf RWC, and photosynthesis were higher in the NaHStreated plants than in the control plants (Figures $\mathbf{1}$ and 3, Supplementary Figure S1). In most plants, the essential goals of 
A

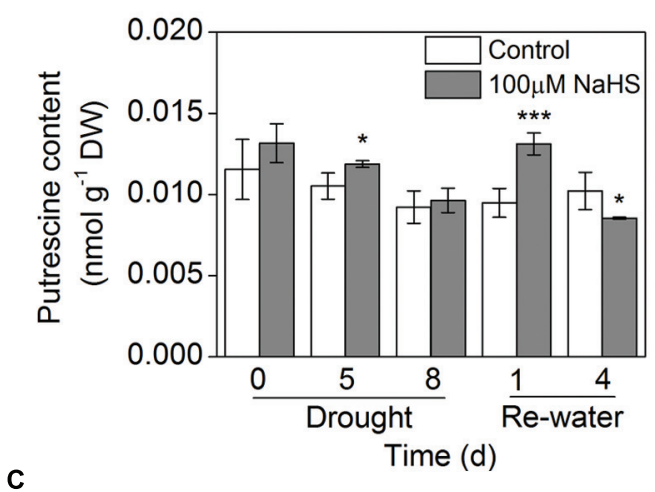

C

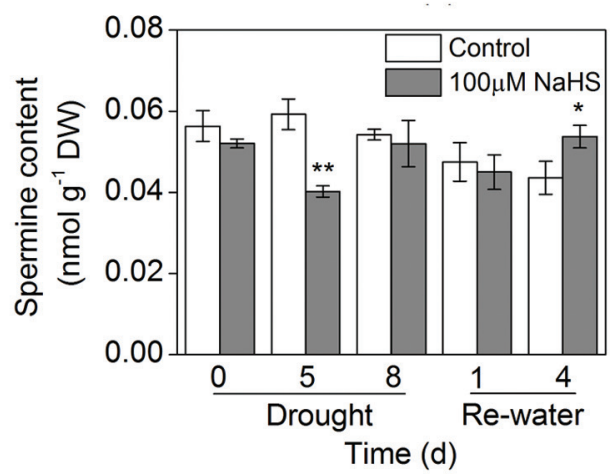

B
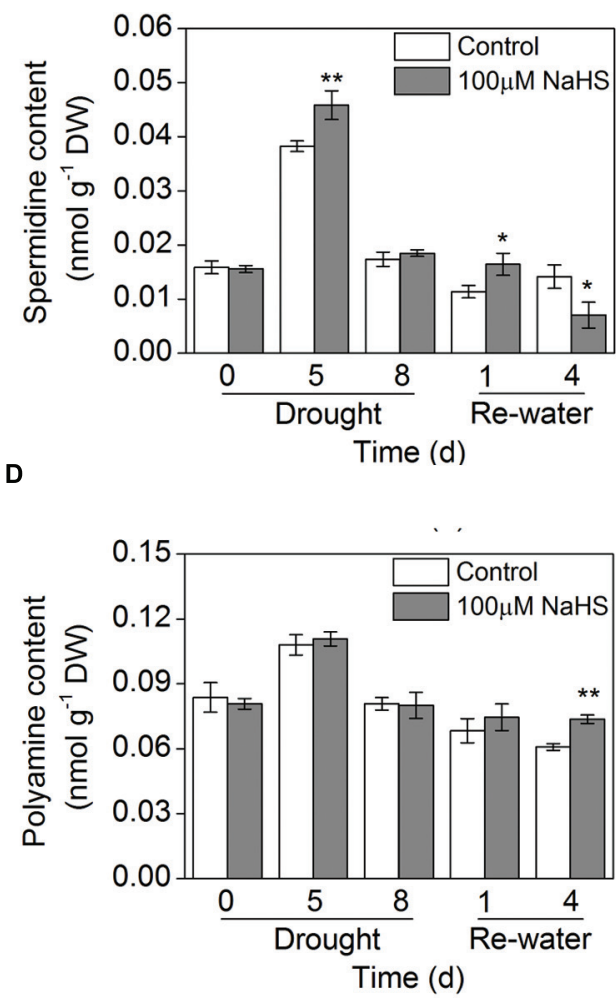

FIGURE 8 | Effect of $\mathrm{H}_{2} \mathrm{~S}$ donor NaHS on free polyamine including putrescine content (A), spermidine content (B), spermine content (C), and the total polyamine content (D) of $\mathbf{6}$-week-old $\mathrm{S}$. oleracea seedlings leaves after drought for $\mathbf{0 , 5}$, and $\mathbf{8} \mathrm{d}$ and rewatering for $\mathbf{1}$ and $\mathbf{4}$ days. The significant level of the difference between control and treatment is indicated by an asterisk $* P<0.05,{ }^{* *} P<0.01$, and ${ }^{* * *} P<0.001$.

drought resistance strategies are to increase water uptake from roots or reduce water loss from the leaves (Yin et al., 2013). In the present study, NaHS-treated plants exhibited significantly less water loss than the control plants (Supplementary Figures S1C,D). Moreover, although the transpiration rate and stomatal conductance were higher in the NaHS-treated plants than in the control plants, the NaHS-treated plants were able to maintain a higher leaf RWC than the control plants, indicating that the alleviation of water stress was not due to the regulation of stomatal closure in this study (Figures $\mathbf{1 C}$ and 3B,D). In this study, under drought stress, the NaHS-treated plants exhibited a high photosynthetic rate compared with the control plants. Under drought stress, the $\mathrm{H}_{2} \mathrm{~S}$ concentration was found that to be higher in NaHS-treated plants than in the untreated plants (Figure 2). These results show that NaHS application enhanced $S$. oleracea seedling resistance to drought stress. Thus, our results indicate that $\mathrm{H}_{2} \mathrm{~S}$ controlled plant the RWC of plant leaves under drought stress. Comparable results have also been observed with silicon, but although silicon alleviated drought stress, the RWC and stomatal conductance were higher in silicon-treated plants than in the untreated plants (Yin et al., 2013). Importantly, in contrast to the above finding, Jin et al. (2013) reported that $\mathrm{H}_{2} \mathrm{~S}$ plays a vital role in the regulation of stomatal closure in the plant response to drought stress. Some possible explanations for this discrepancy include the identity of the plant species, the stress applied, the treatment time and the specific environmental conditions used. The above results showed that $\mathrm{H}_{2} \mathrm{~S}$ can attenuate drought stress in S. oleracea seedlings. However, the precise mechanism by which $\mathrm{H}_{2} \mathrm{~S}$ attenuates drought stress in $S$. oleracea seedlings remains unclear.

\section{$\mathrm{H}_{2} \mathrm{~S}$ Enhances the Tolerance of Plants to Drought Stress by Increasing Osmoprotectants Content}

Under stress condition, plants that have evolved in hightemperature, saline or drought environments usually accumulate three types of osmoprotectants: non-structural sugars and polyols, betaines and allied compounds, and amino acids such as proline. A previous study indicated that salinity decreased cell expansion in tomato plants, which was related to decreases in osmotic and water potentials (Romero-Aranda et al., 2001). These results are consistent with those observed in our experiments (Figures 5A,B). The specific composition of osmoprotectants in plant leaves under drought conditions could be implicated in the specific response observed here. In the present study, the accumulation of soluble sugars, including glucose and sucrose, in the NaHS-treated S. oleracea 
A

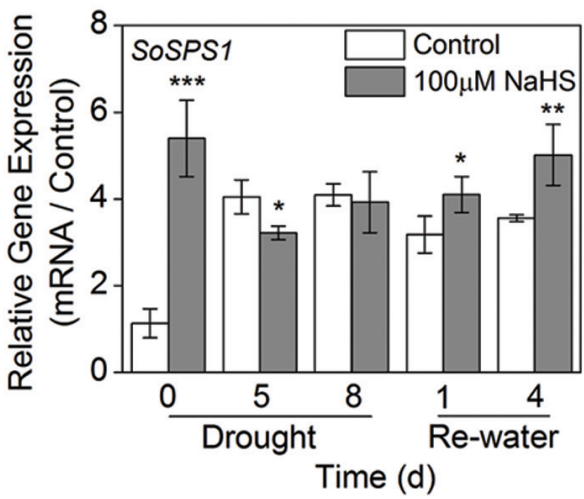

C

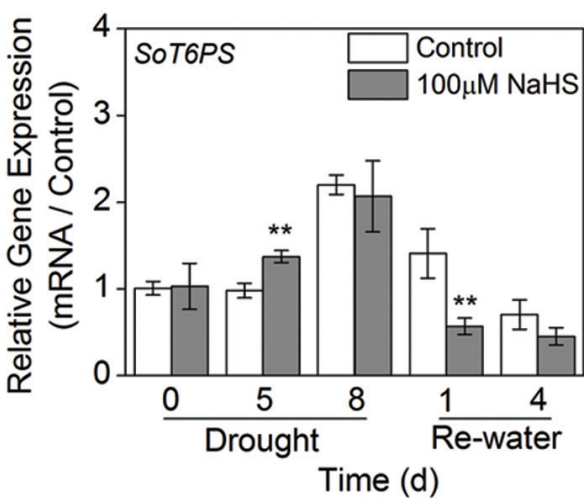

E

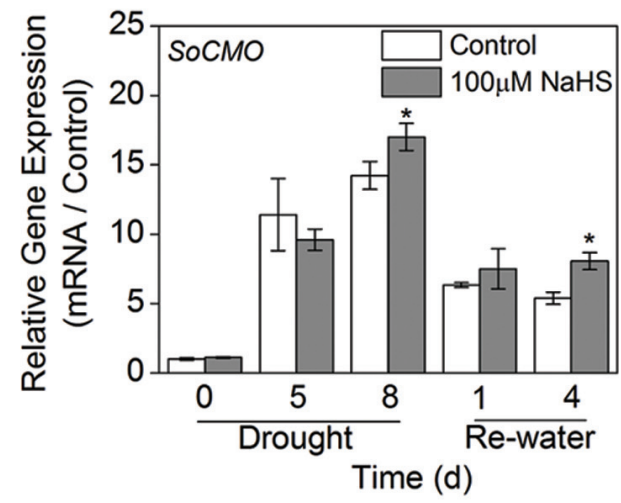

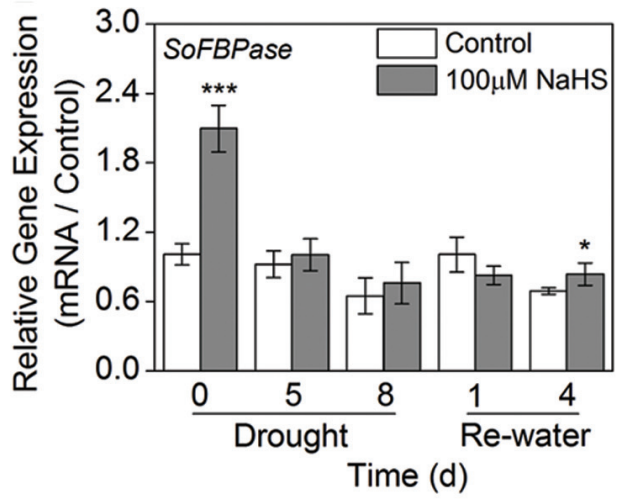

D

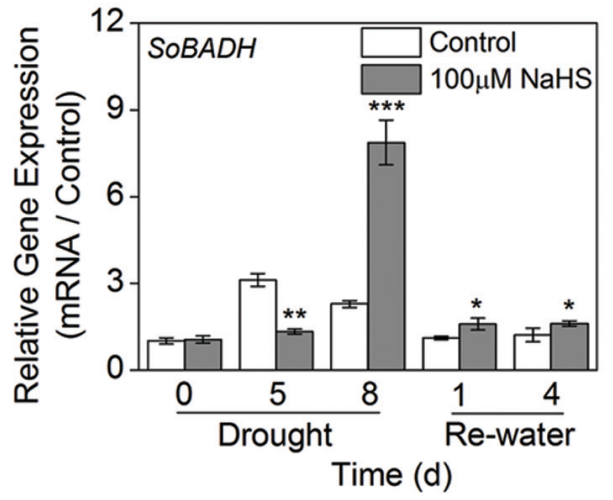

$\mathbf{F}$

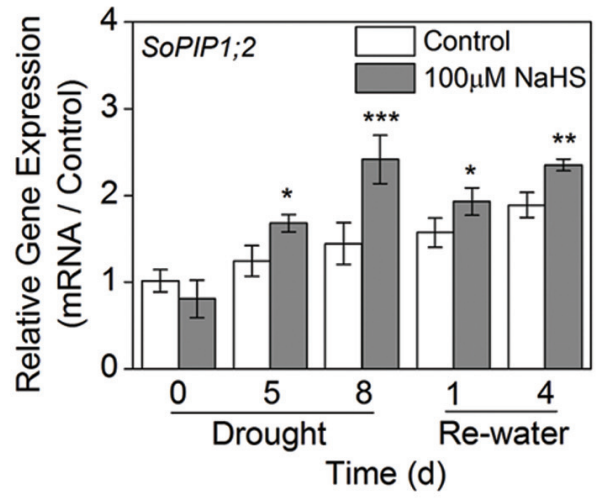

FIGURE 9 | Effect of $\mathrm{H}_{2} \mathrm{~S}$ donor NaHS on the relative transcript abundance of SoSPS1 (A), SoFBPase (B), SoT6PS (C), SoBADH (D), SoCMO (E), and SoPIP1;2 (F) mRNA accumulation in the leaves of $S$. oleracea seedlings after drought for 0,5 , and 8 days and rewatering for 1 and 4 days.

Transcriptional expression of the six genes was measured using real-time qPCR and were normalized with a reference gene (SoGADPH). Mean values \pm SE were calculated from three independent experiments. The significant level of the difference between control and treatment is indicated by an asterisk $* P<0.05$,

${ }^{* *} P<0.01$, and ${ }^{* * *} P<0.001$.

leaves was lower than in the control plants, but the fructose and trehalose contents were significantly increased compared with those of the control plants, suggesting that NaHS enhances drought resistance by regulating the accumulation of soluble sugars, especially the contents of fructose and trehalose (Figure 7). However, under drought and re-watering condition, the expression levels of SoSPS1 and SoFBPase in the NaHS-treated plants increased to varying degrees compared with those in the control plants (Figures 9A,B). Similarly, Rivero et al. (2014) reported that salt and heat treatment specifically up-regulated the expression levels of the FBPase gene. Moreover, the function of this enzyme in the pathway of gluconeogenesis may be a limiting step in the synthesis of starch and trehalose because the activity of FBPase is very important for glucose regeneration, which is 
A

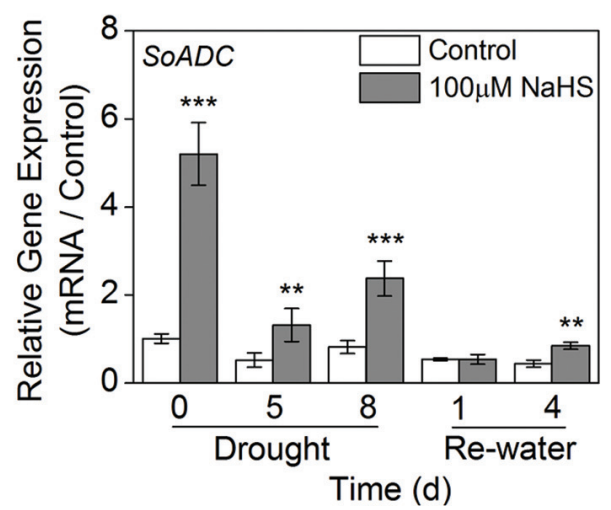

C

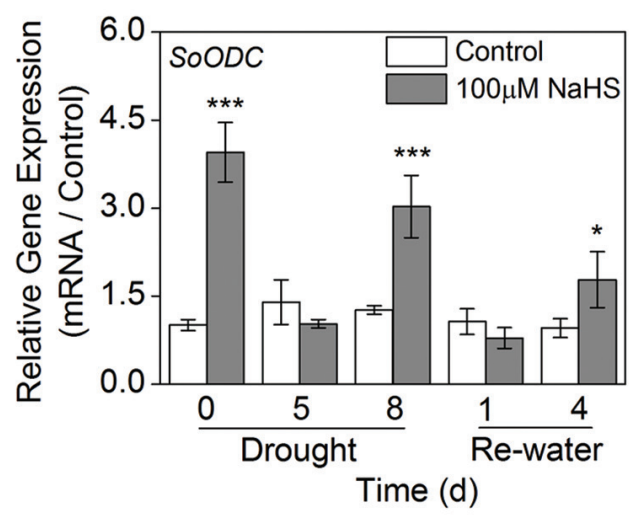

B

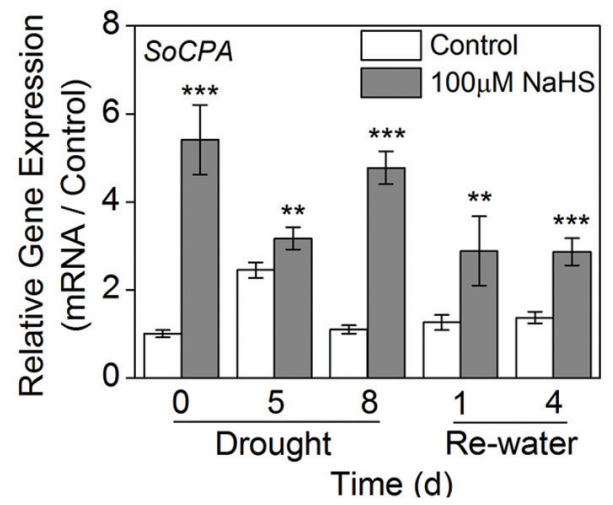

D

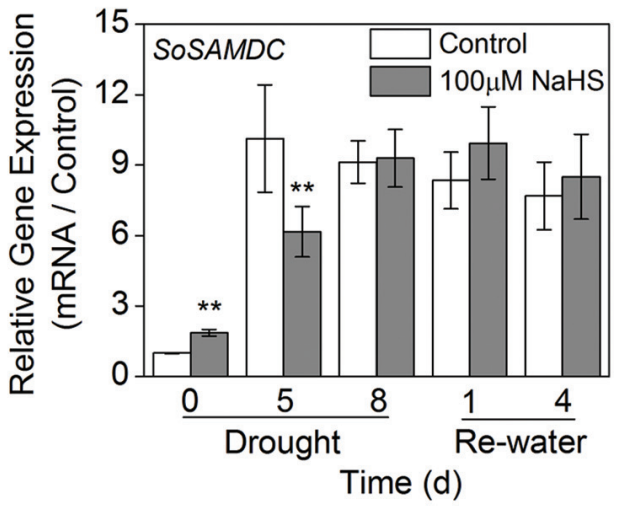

E

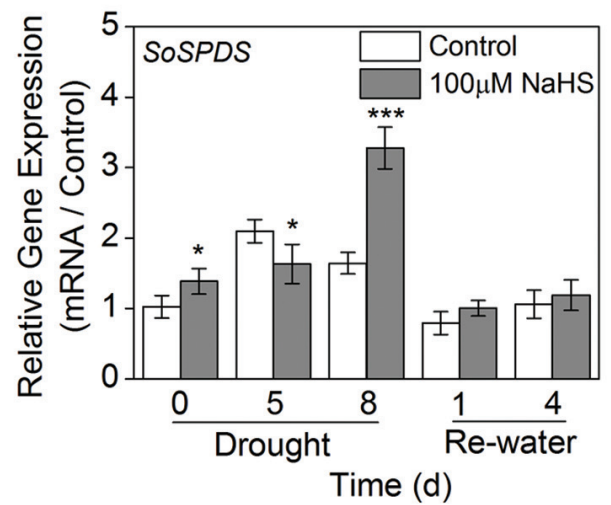

FIGURE 10 | Effect of $\mathrm{H}_{2} \mathrm{~S}$ donor NaHS on the relative transcript abundance of SoADC (A), SoCPA (B), SoODC (C), SoSAMDC (D), and SoSPDS (E) mRNA accumulation in the leaves of $S$. oleracea seedlings after drought for 0,5 , and 8 days and rewatering for $\mathbf{1}$ and 4 days. Transcriptional expression of the six genes was measured using real-time qPCR and were normalized with a reference gene (SoGADPH). Mean values \pm SE were calculated from three independent experiments. The significant level of the difference between control and treatment is indicated by an asterisk ${ }^{*} P<0.05,{ }^{* *} P<0.01$, and ${ }^{* * *} P<0.001$.

the main carbon skeleton in trehalose and starch (Rivero et al., 2014).

Additionally, the function of trehalose in stress response remains controversial, though it has been shown stabilize membranes and protect proteins in desiccated tissues and has been suggested to function as a chemical chaperone (Rivero et al., 2014). However, trehalose levels in nature and even in engineered plants usually remain well below $1 \mathrm{mg} \mathrm{g}^{-1} \mathrm{FW}$, suggesting that trehalose does not act as a compatible solute, but has an alternative function (Garg et al., 2002). The molecular relevance and roles that have been proposed and partially demonstrated for trehalose in plants have been reviewed by Ponnu et al. (2011). Additionally, trehalose-6-phosphate (T6P), a precursor of trehalose, has been proven to play a key role in regulating 


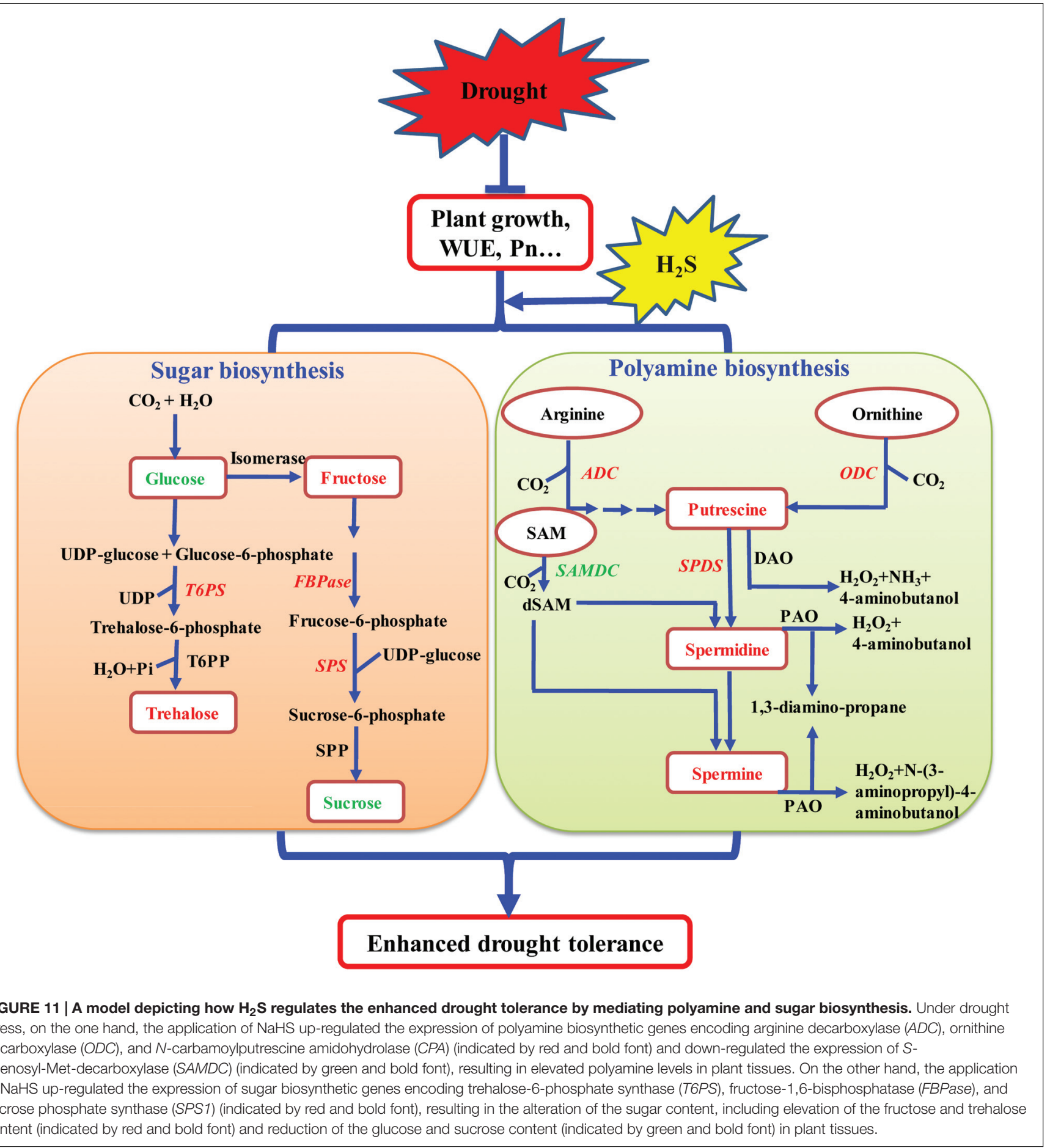

carbohydrate metabolism by inducing starch synthesis in the plastid. Furthermore, trehalose has also been linked to, plant development, cell growth and the induction of photosynthesis capacity. Previous studies have shown that a decrease in the T6P concentration results in the induction of genes involved in photosynthesis-related processes, so that more carbon is used for the growing cell, to supplement the lack of carbon (Ponnu et al., 2011). In the present study, the trehalose content in the
NaHS-treated plants significantly increased after 1 and 4 days of re-watering (Figure 7D). Moreover, the NaHS-treated plants exhibited obvious increases in photosynthesis and PSII efficiency compared with the control plants. Our results show that the increased in photosynthesis capacity and PSII efficiency may also be explained by the regulatory role of trehalose during this process. Furthermore, Garg et al. (2002) suggested that a modulated carbohydrate metabolism could explain the lowered 
photo-oxidative damage associated with the high trehalose levels, which is consistent with our findings.

It is well established that various abiotic stresses such as salinity, drought, cold, and high-temperatures can lead to the accumulation of ROS. Osmoprotective compounds can directly remove ROS or cause the protection of antioxidant enzymes. In this regard, proline has been found to be a singlet oxygen quencher during osmotic stress because it can reduce the damage of ROS in different plants. As an example, the soaking of buds in proline and GB solutions greatly reduces the production of $\mathrm{H}_{2} \mathrm{O}_{2}$, improves the accumulation of soluble sugar and protects the developing tissues from the effects of stress. Similarly, our results also showed that the NaHS-treated plants could accumulate high GB contents and the up-regulated expression of the SoBADH and SoCMO genes under drought conditions (Figures $6 \mathrm{E}$ and 9D,E), which result in lower $\mathrm{H}_{2} \mathrm{O}_{2}$ accumulation and MDA contents in S. oleracea leaves (Figures 6B,C). However, NaHS treatment had no significant effect on the content of proline in S. oleracea seedlings (Figure 6D). A possible explanation for this discrepancy is that proline accumulation results from the activation of its synthesis and an inhibition of its degradation at both the transcriptional and post-transcriptional levels. Therefore, our results indicate that NaHS plays an important role in protecting plants from drought stress by influencing the accumulation of osmoprotective compounds and related gene expression.

\section{$\mathrm{H}_{2} \mathrm{~S}$ Enhances the Tolerance of Plants to Drought Stress by Increasing Polyamine Biosynthesis}

Changes in PA levels and the accumulation of osmoprotective compounds are a common plant response to the lack of water and are associated with plant drought tolerance (Yin et al., 2014). The role of PAs in enhancing drought tolerance has been determined such as increasing the ability of antioxidants, taking effect as stress-signaling regulators, inducing stomatal closure and enhancing leaf water content (Shi et al., 2010; Yin et al., 2014; Muilu-Makela et al., 2015). In addition, the activation of ADC and/or ODC may also stimulate PA biosynthesis in plant tissues because these two enzymes catalyzed the production of Put. Generally, under stress conditions, the pathway of ADC is easily activated; in contrast, ODC is essential for cell proliferation and the biosynthesis of root Put (Tiburcio et al., 2014). Additionally, in this study, the application of NaHS under drought condition resulted in the increased biosynthesis of free and conjugated PAs biosynthesis (Figure 8; Supplementary Figure S2). Moreover, to further study how $\mathrm{H}_{2} \mathrm{~S}$ regulates $\mathrm{PA}$ biosynthesis, the transcriptional abundances of PA biosynthesis-related genes were determined. Our results revealed that the transcription level of SoADC was up-regulated by NaHS under drought condition (Figure 10A). Many previous studies have reported that the ADC pathway is closely related to drought stress and that ADC can regulate the biosynthesis of Put (Yin et al., 2014; Lulai et al., 2015). In fact, the two enzymes agmatine iminohydrolase (AIH) and CPA are also involved in Put biosynthesis in the ADC pathway. Here, the expression level of SoCPA was up-regulated by NaHS (Figure 10B). Interestingly, the transcription levels of SoODC were significantly increased in the NaHS-treated plants grown under drought condition (Figure 10C). Accordingly, these results show that $\mathrm{H}_{2} \mathrm{~S}$ mediated Put production through both the ADC and ODC pathways in S. oleracea seedlings. Additionally, SAMDC is a rate-limiting enzyme in Spd and Spm biosynthesis, because SAMDC has a short half-life. The transcriptional level of $S A M D C$ is induced under different abiotic stresses, and the overexpression of $S A M D C$ increases PA levels and enhances stress tolerance. However, in this study, the transcription of SoSAMDC in the NaHS-treated plants was up-regulated under normal condition, but was down-regulated by NaHS after 5 days of drought, and there was no obvious difference between the treatment periods (Figure 10D). Notably, the Spd level increased markedly after 5 days of drought, whereas the Spm level decreased significantly, suggesting that SAMDC expression did not correlate with the levels of Spd/or Spm, probably as a result of the tight regulation of enzyme activity (Figures 8B,D). However, it is of note that the transcription level of SoSPDS in the NaHStreated plants underwent a significant increase under drought condition (Figure 10E). These results may explain the changes in the Spd and Spm levels observed under drought or re-watering conditions.

Abiotic stress often enhances senescence processes, where valuable resources, accumulated during prior development are recycled for use in other growing parts of the plant. Additionally, previous studies have shown that the increase of PA contents may prevent chlorophyll loss and the inhibition of photosynthesis and thus delay leaf senescence under stress conditions (Shi et al., 2010; Glaubitz et al., 2015). In this study, under drought condition, NaHS was observed to significantly increase photosynthesis in the plants (Figure 3A). Meanwhile, PA contents were also increased to varying degrees, which suggests that PAs may play an important role in the maintenance of photosynthesis and plant growth and additionally may participate in $\mathrm{H}_{2} \mathrm{~S}$-induced drought tolerance. In contrast, if senescence leads to programmed cell death, PAs may play a vital role as a carbon and nitrogen reservoir in plants under stress condition (Moschou et al., 2012). Therefore, PA homoeostasis may be was regulated by $\mathrm{H}_{2} \mathrm{~S}$ to protect plants from drought damage.

\section{Pathway of $\mathrm{H}_{2} \mathrm{~S}$-Induced Drought Resistance}

Based on the above results and the current knowledge concerning the mechanisms of plant response to drought stress, a signaling pathway by which $\mathrm{H}_{2} \mathrm{~S}$ influences the biosynthesis of total PAs and sugar has been proposed. As shown in Figure 11, $\mathrm{H}_{2} \mathrm{~S}$ may promote the growth and photosynthesis of plants under drought stress. Under drought stress, $\mathrm{H}_{2} \mathrm{~S}$ up-regulates the transcription levels of SoADC, SoCPA, SoODC, and SoSPDS, leading to the increased biosynthesis and accumulation of PAs in plant tissues. Under drought stress, $\mathrm{H}_{2} \mathrm{~S}$ may also increase the accumulation of osmoprotective compounds including soluble sugars by changing the expression levels of sugar biosynthesisrelated genes. Together, high levels of PAs and soluble sugars enhance drought tolerance in plants. Our results show that $\mathrm{H}_{2} \mathrm{~S}$ 
regulates drought stress response in plants by mediating certain important metabolic processes and is not limited to a mere mechanical barrier, as was previously assumed. Importantly, our study also opens new avenues of research for the growth and development of plants with enhanced tolerance to abiotic stress conditions.

\section{AUTHOR CONTRIBUTIONS}

JC designed the experiment and wrote the manuscript, Y-TS and $\mathrm{X}-\mathrm{YC}$ conducted the experiment, $\mathrm{W}-\mathrm{HW}$ and E-MH helped in data analysis and presentation, and $\mathrm{ZS}$ and $\mathrm{H}-\mathrm{LZ}$ revised the manuscript.

\section{REFERENCES}

Alcázar, R., Altabella, T., Marco, F., Bortolotti, C., Reymond, M., Koncz, C., et al. (2010a). Polyamines: molecules with regulatory functions in plant abiotic stress tolerance. Planta 231, 1237-1249. doi: 10.1007/s00425-010-1130-0

Alcázar, R., Planas, J., Saxena, T., Zarza, X., Bortolotti, C., Cuevas, J., et al. (2010b). Putrescine accumulation confers drought tolerance in transgenic Arabidopsis plants over-expressing the homologous Arginine decarboxylase 2 gene. Plant Physiol. Biochem. 48, 547-552. doi: 10.1016/j.plaphy.2010.02.002

Alexieva, V., Sergiev, I., Mapelli, S., and Karanov, E. (2001). The effect of drought and ultraviolet radiation on growth and stress markers in pea and wheat. Plant Cell Environ. 24, 1337-1344. doi: 10.1046/j.1365-3040.2001.00778.x

Bates, L. S., Waldren, R. P., and Teare, I. D. (1973). Rapid determination of free proline for water-stress studies. Plant Soil 39, 205-207. doi: 10.1007/ BF00018060

Bessieres, M. A., Gibbon, Y., Lefeuvre, J. C., and Larher, F. (1999). A single-step purification for glycine betaine determination in plant extracts by isocratic HPLC. J. Agric. Food. Chem. 47, 3718-3722. doi: 10.1021/jf990031h

Blum, A. (1996). Crop responses to drought and the interpretation of adaptation. Plant Growth Regul. 20, 135-148. doi: 10.1007/BF00024010

Capell, T., Bassie, L., and Christou, P. (2004). Modulation of the polyamine biosynthetic pathway in transgenic rice confers tolerance to drought stress. Proc. Natl. Acad. Sci. U.S.A. 101, 9909-9914. doi: 10.1073/pnas.0306974101

Chaves, M. M., Maroco, J. P., and Pereira, J. S. (2003). Understanding plant responses to drought - from genes to the whole plant. Funct. Plant Biol. 30, 239-264. doi: 10.1071/FP02076

Chen, J., Wang, W. H., Wu, F. H., He, E. M., Liu, X., Shangguan, Z. P., et al. (2015a). Hydrogen sulfide enhances salt tolerance through nitric oxidemediated maintenance of ion homeostasis in barley seedling roots. Sci. Rep. 5:12516. doi: $10.1038 /$ srep 12516

Chen, J., Wang, W. H., Wu, F. H., You, C. Y., Liu, T. W., Dong, X. J., et al. (2013). Hydrogen sulfide alleviates aluminum toxicity in barley seedlings. Plant Soil 362, 301-318. doi: 10.1007/s11104-012-1275-7

Chen, J., Wu, F. H., Shang, Y. T., Wang, W. H., Hu, W. J., Simon, M., et al. (2015b). Hydrogen sulphide improves adaptation of Zea mays seedlings to iron deficiency. J. Exp. Bot. 66, 6605-6622. doi: 10.1093/jxb/erv368

Chen, J., Wu, F. H., Wang, W. H., Zheng, C. J., Lin, G. H., Dong, X. J., et al. (2011). Hydrogen sulphide enhances photosynthesis through promoting chloroplast biogenesis, photosynthetic enzyme expression, and thiol redox modification in Spinacia oleracea seedlings. J. Exp. Bot. 62, 4481-4493. doi: 10.1093/jxb/ err145

Dhindsa, R. S., Plumb-Dhindsa, P., and Thorpe, T. A. (1981). Leaf senescence: correlated with increased levels of membrane permeability and lipid peroxidation, and decreased levels of superoxide dismutase and catalase. J. Exp. Bot. 32, 93-101. doi: 10.1093/jxb/32.1.93

Duan, J. J., Li, J., Guo, S. R., and Kang, Y. Y. (2008). Exogenous spermidine affects polyamine metabolism in salinity-stressed Cucumis sativus roots and enhances short-term salinity tolerance. J. Plant Physiol. 165, 1620-1635. doi: 10.1016/j.jplph.2007.11.006

\section{ACKNOWLEDGMENTS}

We are grateful to Bing-Bo Li for valuable assistance with the experiments. This study was financially supported by the Natural Science Foundation of China (NSFC) (31501822 and 31401362), the Postdoctoral Science Foundation of China (2015M580876 and 2016T90948).

\section{SUPPLEMENTARY MATERIAL}

The Supplementary Material for this article can be found online at: http://journal.frontiersin.org/article/10.3389/fpls.2016. 01173

Flores, H. E., and Galston, A. W. (1982). Analysis of polyamines in higher plants by high performance liquid chromatography. Plant Physiol. 69, 701-706. doi: 10.1104/pp.69.3.701

García-Mata, C., and Lamattina, L. (2001). Nitric oxide induces stomatal closure and enhances the adaptive plant responses against drought stress. Plant Physiol. 126, 1196-1204. doi: 10.1104/pp.126.3.1196

García-Mata, C., and Lamattina, L. (2010). Hydrogen sulphide, a novel gasotransmitter involved in guard cell signalling. New Phytol. 188, 977-984. doi: 10.1111/j.1469-8137.2010.03465.x

Garg, A. K., Kim, J. K., Owens, T. G., Ranwala, A. P., Choi, Y. D., Kochian, L. V., et al. (2002). Trehalose accumulation in rice plants confers high tolerance levels to different abiotic stresses. Proc. Natl. Acad. Sci. U.S.A. 99, 15898-15903. doi: 10.1073/pnas.252637799

Glaubitz, U., Erban, A., Kopka, J., Hincha, D. K., and Zuther, E. (2015). High night temperature strongly impacts TCA cycle, amino acid and polyamine biosynthetic pathways in rice in a sensitivity-dependent manner. J. Exp. Bot. 66, 6385-6397. doi: 10.1093/jxb/erv352

Hosoki, R., Matsuki, N., and Kimura, H. (1997). The possible role of hydrogen sulfide as an endogenous smooth muscle relaxant in synergy with nitric oxide. Biochem. Biophys. Res. Commun. 237, 527-531. doi: 10.1006/bbrc.1997.6878

Jin, Z., Shen, J., Qiao, Z., Yang, G., Wang, R., and Pei, Y. (2011). Hydrogen sulfide improves drought resistance in Arabidopsis thaliana. Biochem. Biophys. Res. Commun. 414, 481-486. doi: 10.1016/j.bbrc.2011.09.090

Jin, Z., Xue, S., Luo, Y., Tian, B., Fang, H., Li, H., et al. (2013). Hydrogen sulfide interacting with abscisic acid in stomatal regulation responses to drought stress in Arabidopsis. Plant Physiol. Biochem. 62, 41-46. doi: 10.1016/j.plaphy.2012.10.017

Kasinathan, V., and Wingler, A. (2004). Effect of reduced arginine decarboxylase activity on salt tolerance and on polyamine formation during salt stress in Arabidopsis thaliana. Physiol. Plant 121, 101-107. doi: 10.1111/j.00319317.2004.00309.x

Krause, G., and Weis, E. (1991). Chlorophyll fluorescence and photosynthesis: the basics. Annu. Rev. Plant Biol. 42, 313-349. doi: 10.1146/annurev.pp.42.060191. 001525

Li, D., Xiao, Z., Liu, L., Wang, J., Song, G., and Bi, Y. (2010). Effects of exogenous hydrogen sulfide (H2S) on the root tip and root border cells of Pisum sativum. Chin. Bull. Bot. 45, 354-362.

Li, H., Gao, M. Q., Xue, R. L., Wang, D., and Zhao, H. J. (2015). Effect of hydrogen sulfide on D1 protein in wheat under drought stress. Acta Physiol. Plant 37, 1-9. doi: 10.1007/s11738-015-1975-8

Li, L., Bhatia, M., and Moore, P. K. (2006). Hydrogen sulphide - a novel mediator of inflammation? Curr. Opin. Pharmacol. 6, 125-129. doi: 10.1016/j.coph.2005.10.007

Livak, K. J., and Schmittgen, T. D. (2001). Analysis of relative gene expression data using teal-time quantitative PCR and the $2^{-\Delta \Delta C t}$ method. Methods 25, 402-408. doi: 10.1006/meth.2001.1262

Lulai, E. C., Neubauer, J. D., Olson, L. L., and Suttle, J. C. (2015). Wounding induces changes in tuber polyamine content, polyamine metabolic gene expression, and enzyme activity during closing layer formation 
and initiation of wound periderm formation. J. Plant Physiol. 176, 89-95. doi: 10.1016/j.jplph.2014.12.010

Moschou, P. N., Wu, J., Cona, A., Tavladoraki, P., Angelini, R., and RoubelakisAngelakis, K. A. (2012). The polyamines and their catabolic products are significant players in the turnover of nitrogenous molecules in plants. J.Exp. Bot. 63, 5003-5015. doi: 10.1093/jxb/ers202

Muilu-Makela, R., Vuosku, J., Laara, E., Saarinen, M., Heiskanen, J., Haggman, H., et al. (2015). Water availability influences morphology, mycorrhizal associations, PSII efficiency and polyamine metabolism at early growth phase of Scots pine seedlings. Plant Physiol. Biochem. 88, 70-81. doi: 10.1016/j.plaphy.2015.01.009

Peeva, V., and Cornic, G. (2009). Leaf photosynthesis of Haberlea rhodopensis before and during drought. Environ. Exp. Bot. 65, 310-318. doi: 10.1016/j.envexpbot.2008.09.009

Ponnu, J., Wahl, V., and Schmid, M. (2011). Trehalose-6-Phosphate: connecting plant metabolism and development. Front. Plant Sci. 2:70. doi: 10.3389/fpls.2011.00070

Prioul, J. L., and Chartier, P. (1977). Partitioning of transfer and carboxylation components of intracellular resistance to photosynthetic $\mathrm{CO} 2$ fixation: a critical analysis of the methods used. Ann. Bot. 41, 789-800.

Rivero, R. M., Mestre, T. C., Mittler, R. O. N., Rubio, F., Garcia-Sanchez, F., and Martinez, V. (2014). The combined effect of salinity and heat reveals a specific physiological, biochemical and molecular response in tomato plants. Plant Cell Environ. 37, 1059-1073. doi: 10.1111/pce.12199

Romero-Aranda, R., Soria, T., and Cuartero, J. (2001). Tomato plant-water uptake and plant-water relationships under saline growth conditions. Plant Sci. 160, 265-272. doi: 10.1016/S0168-9452(00)00388-5

Sekiya, J., Schmidt, A., Wilson, L. G., and Filner, P. (1982). Emission of hydrogen sulfide by leaf tissue in response to L-cysteine. Plant Physiol. 70, 430-436. doi: 10.1104/pp.70.2.430

Shan, C. J., Zhang, S. L., Li, D. F., Zhao, Y. Z., Tian, X. L., Zhao, X. L., et al. (2011). Effects of exogenous hydrogen sulfide on the ascorbate and glutathione metabolism in wheat seedlings leaves under water stress. Acta Physiol. Plant 33, 2533-2540. doi: 10.1007/s11738-011-0746-4

Shi, J., Fu, X. Z., Peng, T., Huang, X. S., Fan, Q. J., and Liu, J. H. (2010). Spermine pretreatment confers dehydration tolerance of citrus in vitro plants via modulation of antioxidative capacity and stomatal response. Tree Physiol. 30, 914-922. doi: 10.1093/treephys/tpq030

Takahashi, T., and Kakehi, J. I. (2010). Polyamines: ubiquitous polycations with unique roles in growth and stress responses. Ann. Bot. 105, 1-6. doi: $10.1093 / \mathrm{aob} / \mathrm{mcp} 259$

Tiburcio, A., Altabella, T., Bitrián, M., and Alcázar, R. (2014). The roles of polyamines during the lifespan of plants: from development to stress. Planta 240, 1-18. doi: 10.1007/s00425-014-2055-9

Toumi, I., Moschou, P. N., Paschalidis, K. A., Bouamama, B., Ben Salem-fnayou, A., Ghorbel, A. W., et al. (2010). Abscisic acid signals reorientation of polyamine metabolism to orchestrate stress responses via the polyamine exodus pathway in grapevine. J. Plant Physiol. 167, 519-525. doi: 10.1016/j.jplph.2009.10.022

Tun, N. N., Santa-Catarina, C., Begum, T., Silveira, V., Handro, W., Floh, E. I. S., et al. (2006). Polyamines induce rapid biosynthesis of nitric oxide (NO) in Arabidopsis thaliana seedlings. Plant Cell Physiol. 47, 346-354. doi: 10.1093/pcp/pci252

Wang, A. G., and Luo, G. H. (1990). Quantitative relation between the reaction of hydroxylamine and superoxide anion radicals in plants. Plant Physiol. Commun. 84, 2895-2898.

Wang, B. L., Shi, L., Li, Y. X., and Zhang, W. H. (2010). Boron toxicity is alleviated by hydrogen sulfide in cucumber (Cucumis sativus L.) seedlings. Planta 231, 1301-1309. doi: 10.1007/s00425-010-1134-9

Wang, R. (2002). Two's company, three's a crowd: can H2S be the third endogenous gaseous transmitter? FASEB J. 16, 1792-1798. doi: 10.1096/fj.02-0211hyp

Wang, Y., Li, L., Cui, W., Xu, S., Shen, W., and Wang, R. (2012). Hydrogen sulfide enhances alfalfa (Medicago sativa) tolerance against salinity during seed germination by nitric oxide pathway. Plant Soil 351, 107-119. doi: 10.1007/s11104-011-0936-2

Wen, X. P., Pang, X. M., Matsuda, N., Kita, M., Inoue, H., Hao, Y. J., et al. (2008). Over-expression of the apple spermidine synthase gene in pear confers multiple abiotic stress tolerance by altering polyamine titers. Transgenic Res. 17, 251-263. doi: $10.1007 /$ s11248-007-9098-7
Whittaker, J. B. (1994). Physiological responses of leaves of Rumex obtusifolius to damage by a leaf miner. Funct. Ecol. 8, 627-630. doi: 10.2307/2389925

Wilkinson, S., and Davies, W. J. (2010). Drought, ozone, ABA and ethylene: new insights from cell to plant to community. Plant Cell Environ. 33, 510-525. doi: 10.1111/j.1365-3040.2009.02052.x

Yamasaki, H., and Cohen, M. F. (2006). NO signal at the crossroads: polyamineinduced nitric oxide synthesis in plants? Trends Plant Sci. 11, 522-524. doi: 10.1016/j.tplants.2006.09.009

Yang, G., Wu, L., Jiang, B., Yang, W., Qi, J., Cao, K., et al. (2008). H2S as a physiologic vasorelaxant: hypertension in mice with deletion of cystathionine $\gamma$-Lyase. Science 322, 587-590. doi: 10.1126/science.1162667

Yin, L. N., Wang, S. W., Li, J. Y., Tanaka, K., and Oka, M. (2013). Application of silicon improves salt tolerance through ameliorating osmotic and ionic stresses in the seedling of Sorghum bicolor. Acta Physiol. Plant 35, 3099-3107. doi: 10.1007/s11738-013-1343-5

Yin, L. N., Wang, S. W., Liu, P., Wang, W. H., Cao, D., Deng, X. P., et al. (2014). Silicon-mediated changes in polyamine and 1-aminocyclopropane-1carboxylic acid are involved in silicon-induced drought resistance in Sorghum bicolor L. Plant Physiol.Biochem. 80, 268-277. doi: 10.1016/j.plaphy.2014.04.014

Yin, L. N., Wang, S. W., Tanaka, K., Fujihara, S., Itai, A., Deng, X. P., et al. (2015). Silicon-mediated changes in polyamines participate in siliconinduced salt tolerance in Sorghum bicolor L. Plant Cell Environ. 39, 245-258. doi: 10.1111/pce.12521

Yoo, C. Y., Pence, H. E., Jin, J. B., Miura, K., Gosney, M. J., Hasegawa, P. M., et al. (2010). The Arabidopsis GTL1 transcription factor regulates water use efficiency and drought tolerance by modulating stomatal density via transrepression of SDD1. Plant Cell 22, 4128-4141. doi: 10.1105/tpc.110.078691

Zhang, H., Hu, L. Y., Hu, K. D., He, Y. D., Wang, S. H., and Luo, J. P. (2008). Hydrogen sulfide promotes wheat seed germination and alleviates oxidative damage against copper stress. J. Integr. Plant Biol. 50, 1518-1529. doi: 10.1111/j.1744-7909.2008.00769.x

Zhang, H., Hu, S. L., Zhang, Z. J., Hu, L. Y., Jiang, C. X., Wei, Z. J., et al. (2011). Hydrogen sulfide acts as a regulator of flower senescence in plants. Postharvest Biol. Technol. 60, 251-257. doi: 10.1016/j.postharvbio.2011.01.006

Zhang, H., Jiao, H., Jiang, C. X., Wang, S. H., Wei, Z. J., Luo, J. P., et al. (2010a). Hydrogen sulfide protects soybean seedlings against drought-induced oxidative stress. Acta Physiol. Plant 32, 849-857. doi: 10.1007/s11738-010-0469-y

Zhang, H., Tan, Z. Q., Hu, L. Y., Wang, S. H., Luo, J. P., and Jones, R. L. (2010b). Hydrogen sulfide alleviates aluminum toxicity in germinating wheat seedlings. J. Integr. Plant Biol. 52, 556-567. doi: 10.1111/j.1744-7909.2010. 00946.x

Zhang, H., Ye, Y. K., Wang, S. H., Luo, J. P., Tang, J., and Ma, D. F. (2009). Hydrogen sulfide counteracts chlorophyll loss in sweetpotato seedling leaves and alleviates oxidative damage against osmotic stress. Plant Growth Regul. 58, 243-250. doi: 10.1007/s10725-009-9372-1

Zhao, T. Y., Thacker, R., Corum, J. W., Snyder, J. C., Meeley, R. B., Obendorf, R. L., et al. (2004). Expression of the maize GALACTINOL SYNTHASE gene family: (I) Expression of two different genes during seed development and germination. Physiol. Plant 121, 634-646. doi: 10.1111/j.1399-3054.2004.00367.x

Zhao, W., Zhang, J., Lu, Y., and Wang, R. (2001). The vasorelaxant effect of H2S as a novel endogenous gaseous KATP channel opener. EMBO J. 20, 6008-6016. doi: 10.1093/emboj/20.21.6008

Ziogas, V., Tanou, G., Belghazi, M., Filippou, P., Fotopoulos, V., Grigorios, D., et al. (2015). Roles of sodium hydrosulfide and sodium nitroprusside as priming molecules during drought acclimation in citrus plants. Plant Mol. Biol. 89, 433-450. doi: 10.1007/s11103-015-0379-x

Conflict of Interest Statement: The authors declare that the research was conducted in the absence of any commercial or financial relationships that could be construed as a potential conflict of interest.

Copyright (c) 2016 Chen, Shang, Wang, Chen, He, Zheng and Shangguan. This is an open-access article distributed under the terms of the Creative Commons Attribution License (CC BY). The use, distribution or reproduction in other forums is permitted, provided the original author(s) or licensor are credited and that the original publication in this journal is cited, in accordance with accepted academic practice. No use, distribution or reproduction is permitted which does not comply with these terms. 